Pacific Journal of Mathematics

REMARKS ON DE LA VALLÉE POUSSIN MEANS AND
CONVEX CONFORMAL MAPS OF THE CIRCLE G. PÓLYA AND I. J. SCHOENBE 


\title{
REMARKS ON DE LA VALLÉE POUSSIN MEANS \\ AND CONVEX CONFORMAL MAPS \\ OF THE CIRCLE
}

\author{
G. Pólya and I. J. Schoenberg
}

Introduction. The aims of the present remarks are similar to those pursued by L. Fejér in several papers in the early nineteen thirties and well described by the title of one of his paper: Gestaltliches über die Partialsummen und ihre Mittelwerte bei der Fourierreihe und der Potenzreihe. However, the means which we use to realize these aims are different. Fejér discovered the remarkable behavior of certain Cesàro means, especially that of the third Cesàro means for even or odd functions of certain simple basic shapes. In what follows we show that the de la Vallée Poussin means possess such shape-preserving properties to a much higher degree thanks to their variation diminishing character.

Before stating our results, we have to explain a few concepts.

Variation diminishing Transformations on the Circle. If $a_{1}, a_{2}, \cdots, a_{n}$ $\mathrm{s}$ a finite sequence of real numbers we shall denote by $v(\alpha)$ or $v\left(a_{\nu}\right)$ the number of variations of sign in the terms of this sequence. By the number $v_{c}(a)$ of cyclic variations of sign of our sequence we mean the following: If all $a_{\nu}=0$ we set $v_{c}(a)=0$. If $\alpha_{i} \neq 0$ we set

$$
v_{c}(a)=v\left(a_{i}, a_{i+1}, \cdots, a_{n}, a_{1}, a_{2}, \cdots, a_{i-1}, a_{i}\right) .
$$

If we think of the $a_{\nu}$ as arranged clockwise in cyclic order, it becomes obvious that $v_{c}(a)$ does not depend on the particular non-vanishing term $a_{i}$ we start with. Notice that $v_{c}(a)$ is always an even number. Let now $f(t)$ be a real-valued function of period $2 \pi$. Let $t_{1}, t_{2}, \cdots, t_{n}$ be such that

$$
t_{1}<t_{2}<\cdots<t_{n}<t_{1}+2 \pi \text {. }
$$

We may now define the number $v_{c}(f)$ of cyclic variations of sign of $f(t)$ by

$$
v_{c}(f)=\sup v_{c}\left(f\left(t_{\nu}\right)\right)
$$

the supremum being taken for all finite sequences $\left\{t_{\nu}\right\}$ subject to (1).

Received October 21, 1957. This paper was prepared partly under the sponsorship of the Office of Naval Research, and partly under the sponsorship of the United States Air Force, Office of Scientific Research, Air Research and Development Command. 
Also $v_{c}(f)$, if finite, is even. Thus $v_{c}(\sin t)=2, v_{c}(\sin 2 t)=4, v_{c}(|\sin t|)=0$.

We now describe what is meant by a variation diminishing transformation on the circle (See [4]). Such a transformation is characterized by a non-negative weight-function, or kernel, $\Omega(t)$, of period $2 \pi$, of bounded variation and normalized by the conditions

$$
\frac{1}{2 \pi} \int_{0}^{2 \pi} \Omega(t) d t=1, \Omega(t)=\frac{1}{2}(\Omega(t+0)+\Omega(t-0)) .
$$

Let $f(t)$ be an arbitrary periodic function, with period $2 \pi$, real-valued and integrable (cf. §1.2); let us form its convolution transform

$$
g(t)=\frac{1}{2 \pi} \int_{0}^{2 \pi} \Omega(t-\tau) f(\tau) d \tau
$$

We say that this transformation is variation diminishing provided that the inequality

$$
v_{c}(g) \leqq v_{c}(f)
$$

holds for each $f$. We mean the same thing if we say that $\Omega(t)$ is a variation diminishing kernel.

$V$-means. One of our aims is to show that the de la Vallée Poussin kernels

$$
\omega_{n}(t)=\frac{(n !)^{2}}{(2 n) !}\left(2 \cos \frac{t}{2}\right)^{2 n}
$$

the Fourier expansion of which has the simple form

$$
\omega_{n}(t)=\frac{1}{\left(\begin{array}{c}
2 n \\
n
\end{array}\right)} \sum_{-n}^{n}\left(\begin{array}{l}
2 n \\
n+\nu
\end{array}\right) e^{i \nu t}=1+2 \sum_{1}^{n} \frac{n !}{(n-\nu) !} \frac{n !}{(n+\nu) !} \cos \nu t,
$$

possess the property of being variation diminishing for $n=1,2,3, \cdots$. For $\Omega(t)=\omega_{n}(t)$ the transformation (4) becomes

$$
V_{n}(t)=\frac{(n !)^{2}}{(2 n) !} \frac{1}{2 \pi} \int_{0}^{2 \pi}\left(2 \cos \frac{t-\tau}{2}\right)^{2 n} f(\tau) d \tau
$$

and defines the de la Vallée Poussin means, or simply $V$-means, of the function $f(t)$. It is easily verified (See [14] and [5, p. 15]) that $V_{n}(t)$ is a trigonometric polynomial of an order not exceeding $n$, which is readily expressed in terms of the Fourier coefficients of $f(t)$. Indeed, if

$$
f(t) \sim \sum_{-\infty}^{\infty} c_{\nu} e^{i \nu t},\left(c_{-\nu}=\bar{c}_{\nu}\right),
$$


we obtain by convoluting $\left(6^{\prime}\right)$ and $(8)$

$$
V_{n}(t)=\frac{1}{\left(\begin{array}{c}
2 n \\
n
\end{array}\right)} \sum^{n}\left(\begin{array}{l}
2 n \\
n+\nu
\end{array}\right) c_{2} e^{i \nu t}
$$

In terms of the real Fourier series $\left(2 c_{\nu}=a_{\nu}-i b_{\nu}\right)$

$$
f(t) \sim{ }_{2}^{1} a_{0}+\sum_{1}^{\infty}\left(a_{\nu} \cos \nu t+b_{\nu} \sin \nu t\right)
$$

we find

$$
V_{n}(t)=\frac{1}{2} a_{\nu}+\frac{1}{\left(\begin{array}{c}
2 n \\
n
\end{array}\right)} \sum_{1}^{n}\left(\begin{array}{l}
2 n \\
n+\nu
\end{array}\right)\left(a_{\nu} \cos \nu t+b_{\nu} \sin \nu t\right)
$$

or

$$
V_{n}(t)=\frac{1}{2} a_{0}+\sum_{1}^{n} \frac{n !}{(n-\nu) !} \frac{n !}{(n-\nu) !}\left(a, \cos \nu t+b_{\nu} \sin \nu t\right) .
$$

Main Results. Our principal result is the following

\section{THEOREM 1. The inequalities}

$$
v_{c}\left(V_{n}\right) \leqq Z_{c}\left(V_{n}\right) \leqq v_{c}(f)
$$

hold for an arbitrary integrable function $f(t)$. (We let $Z_{c}\left(V_{n}\right)$ denote the number of real zeros of $V_{n}(t)$ within a period including multiplicities.)

The first inequality $v_{c}\left(V_{n}\right) \leqq Z_{c}\left(V_{n}\right)$, which is obvious, shows that Theorem 1 states considerably more than the variation diminishing property of the kernel $\omega_{n}(t)$ which amounts to $v_{c}\left(V_{n}\right) \leqq v_{c}(f)$. In Part I we give two proofs of Theorem 1, both based on a theorem due to Sylvester [12]. The first proof uses the result of Sylvester's theorem, the second uses the method of one of its proofs.

In Part II we discuss applications of the variation diminishing property of $V$-means. Theorem 1 gives a useful lower bound for $v_{c}(f)$ if a certain number of Fourier coefficients of $f(t)$ are known. It is shown how this implies easily some results by Sturm, A. Hurwitz, Pólya and Wiener. In $\$ 5$ we study the simplest classes of discontinuous periodic functions; the behavior of their $V$-means is described by Theorems 3 and 4. Fejér's Theorem III $[1, p$. 86] has an analogue for $V$-means which is our Theorem 5 below. All this refers to real periodic functions. However, the shape-preserving properties of $V$-means appear to best advantage if applied to complex-valued periodic functions. 
Let us state here the main result of $\S 6$ concerning convex maps of the circle. Let $K$ denote the class of those "schlicht" power series $\sum_{1}^{\infty} a$. $z^{\nu}$ which map $|z|<1$ onto some convex domain. Let

$$
\begin{array}{r}
f(z)=\sum_{1}^{\infty} c_{2} z^{\nu},\left(c_{1}=1\right), \\
\boldsymbol{V}_{n}(z)=\frac{1}{\left(\begin{array}{c}
2 n \\
n
\end{array}\right)} \sum_{\nu=1}^{n}\left(\begin{array}{l}
2 n \\
n+\nu
\end{array}\right) c_{2} z^{\nu}
\end{array}
$$

be the de la Vallée Poussin mean, or $\boldsymbol{V}$-mean, of the power series (14). It is known that the partial sums of the series (14) need not belong to $K$. G. Szegö has shown [13] that if $F(z) \in K$ then all partial sums of (14) are "schlicht" in the circle $|z|<1 / 4$ and map it onto convex domains, and that $1 / 4$ is here the largest constant. That the $V$-means belong to $K$ is one part of the following

ThEOREM 2. For

$$
f(z) \in K
$$

it is necessary and sufficient that

$$
\boldsymbol{V}_{n}(z) \in K \text { for } n=1,2, \cdots \text {. }
$$

The sufficiency part does not even assume the regularity of (14) in the unit circle, as for any formal power series (14) the assumption (17) imply that (14) converges and defines an element of $K$.

Additional Results. Parts I and II are followed by two appendices which contain related materials, but are almost independent of the main text.

Appendix I brings out a certain analogy between approximations to two kinds of functions: periodic functions and functions defined in a finite interval. It will be shown that the shape-preserving properties of the $V$-means, which approximate functions of period $2 \pi$, are analogous to the shape-preserving properties of the so called Bernstein polynomials which approximate functions defined in $[0,1]$. For the definition of these polynomials see $\$ 7$ where also their variation diminishing property (Theorem 6) is stated and proved.

Appendix II is devoted to a conjecture on power series which represent a conformal one to one mapping of the unit circle onto a convex domain. The conjecture is that the Hadamard composition, or convolution, of two such power series is again a power series of the same kind (see §9). We do not know whether this conjecture is true or not (it seems to us more likely that it is true) but at any rate, in view of the 
partial results which we have obtained ( $\$ 10$ and 11$)$, the problem to prove or to disprove the conjecture seems to us worth while.

\section{PART I. THE DE LA VALLÉE POUSSIN SUMMATION METHOD IS VARIATION DIMINISHING}

1.1 A theorem of Sylvester. In the course of his work on Newton's rule of signs J. J. Sylvester discovered a remarkable theorem concerning the real zeros of polynomials of the form

$$
\sum_{\nu=1}^{m} c_{\nu}\left(x-\xi_{\nu}\right)^{q}
$$

(see [12, p. 408], [7] and also [9, vol. 2, Problem 79, p. 50]). In Sylvester's theorem $q$ may assume any positive integral value, a fact which is important for its proof which proceeds by induction in $q$. We need Sylvester's result only for $q=2 n$ and state it as follows.

Lemma 1. Let $\xi_{1}<\xi_{2}<\cdots<\xi_{m},(m \geqq 2)$, be given reals and consider the polynomial

$$
P(x)=\sum_{\nu=1}^{m} c_{\nu}\left(x-\xi_{\nu}\right)^{2 n}
$$

(with real $c_{\nu} \neq 0$ for all $\nu$ ), which we assume not to vanish identically. Then

$$
Z(P ;-\infty<x<\infty) \leqq v\left(c_{1}, c_{2}, \cdots, c_{m}, c_{1}\right),
$$

where the left side denotes the number of real zeros of $P(x)$ while the right side is the number of variations of sign in the sequence displayed.

The significance for us of Sylvester's results is that it easily yields the following

LEMMA 2. Let

$$
-\pi<\tau_{1}<\tau_{2}<\cdots<\tau_{m}<\pi, \quad m \geqq 2,
$$

be given reals and consider the trigonometric polynomial

$$
T(t)=\sum_{\nu=1}^{m} c_{\nu}\left(\sin \frac{t-\tau_{\nu}}{2}\right)^{2 n},
$$

(for real $c_{\nu} \neq 0$ for all $\nu$ ), which we assume not to vanish identically. Then

$$
Z(T ;-\pi<t<\pi) \leqq v\left(c_{1}, c_{2}, \cdots, c_{m}, c_{1}\right) .
$$

Proof. We introduce the new variable 


$$
x=\tan \frac{t}{2}
$$

whose range is $-\infty<x<\infty$. The images of the $\tau_{\nu}$ we denote by

$$
\xi_{\nu}=\tan \frac{\tau_{\nu}}{2}
$$

and these give rise to the identities

$$
\left(\sin \frac{t-\tau_{\nu}}{2}\right)^{2}=\frac{\left(x-\xi_{\nu}\right)^{2}}{\left(1+x^{2}\right)\left(1+\xi_{\nu}^{2}\right)}, \quad \nu=1, \cdots, m,
$$

Thus (1.2) may be expressed in terms of $x$ by

$$
T(t)=\frac{1}{\left(1+x^{2}\right)^{n}} \sum_{\nu=1}^{m} c_{\nu} r_{2}\left(x-\xi_{\nu}\right)^{2 n},
$$

where the $\gamma_{\nu}$ are positive and so Lemma 2 immediately follows from Sylvester's Lemma 1.

We now recast our result in the following more useful form;

LEMma 3. Let $\tau_{1}, \tau_{2}, \cdots, \tau_{m}(m \geqq 2)$ be $m$ points in counter-clockwise order on the circle such that $\tau_{m}$ should not overtake or even reach $\tau_{1}$. We may express these requirements by assuming that

$$
\tau_{1}<\tau_{2}<\cdots<\tau_{m}<\tau_{1}+2 \pi
$$

Let

$$
T_{n}(t)=\sum_{\nu=1}^{m} c_{\imath} \omega_{n}\left(t-\tau_{\nu}\right)
$$$$
T_{n}(t) \not \equiv 0,
$$

where at least two among the $c_{\nu}$ do not vanish. Then

$$
Z_{c}\left(T_{n}\right) \leqq v_{c}\left(c_{\nu}\right) \text {. }
$$

Proof. By omitting vanishing terms in (1.5) we may assume that $c_{\nu} \neq 0$ for all $\nu$. Moreover, a change of variable by $t=t^{\prime}-\pi$ will evidently not alter the left hand side of (1.6). This implies that in our statement (1.6) we may replace $T_{n}(t)$ by the polynomial $T(t)$ defined by (1.2). By a second appropriate transformation $t=t^{\prime}+c$ we may replace the conditions (1.4) by the more restrictive inequalities (1.1), at the same time making sure that $T(\pi) \neq 0$. But then

$$
Z_{c}\left(T_{n}\right)=Z_{c}(T)=Z(T ;-\pi<t<\pi) \leqq v\left(c_{1}, c_{2}, \cdots, c_{m} . c_{\eta}\right)=v_{c}\left(c_{\nu}\right)
$$

and Lemma 3 is established. 
1.2. On the number of variations of a function. The reader may interpret the term "integrable" either according to the definition of Riemann or to that of Lebesgue, or to any other definition that involves the familiar standard properties of the integral. We emphasize the following property: If $f(t)$ and $g(t)$ are integrable and $f(t) \geqq 0$ in the interval $I$, then

$$
\int_{I} f d t=0
$$

implies

$$
\int_{I} f g d t=0 \text {. }
$$

We consider now a real-valued periodic function $f(t)$ with the period $2 \pi$, we assume that it is integrable in the interval $(0,2 \pi)$ and that $v_{c}(f)$, as defined in the Introduction, is finite. We consider $t(\bmod 2 \pi)$, that is, we consider $t$ as attached to a point on the periphery of the unit circle. If $v_{c}(f)=2 k$, we can, as easily seen, divide the circumference of the unit circle into $2 k$ consecutive ares

$$
I_{1}, I_{2}, \cdots, I_{2 k}
$$

such that

$$
(-1)^{\nu-1} f(t) \geqq 0 \text { in } I_{\nu}
$$

for $\nu=1,2, \cdots, 2 k$; the arcs (1.7) may be open, or closed, or open from one side and closed from the other, some of them may even reduce to a single point. Now, we normalize $f(t)$, that is, we change $f(t)$ (if necessary) as follows: we set $f(t)=0$ in all points of any interval (1.7) on which $\int f d t$ vanishes ; especially, if an interval listed under (1.7) consists of just one point, we set $f(t)=0$ in that point. This normalization cannot increase (but may decrease) $v_{c}(f)$ and leaves unchanged the $V$ means of $f$ (cf. the initial remark of this section). Therefore, it will be sufficient to prove Theorem 1 for normalized functions. If, however, $v_{c}(f)=2 k$ for a normalized $f(t)$, the intervals (1.7), constructed as above, have the property

$$
(-1)^{\nu-1} \int_{I_{\nu}} f(t) d t>0 \text { for } \nu=1,2, \cdots, 2 k \text {. }
$$

The foregoing remarks will be useful in the following proof of Theorem 1. Yet we do not need them in establishing the weaker inequality

$$
v_{c}\left(V_{n}\right) \leqq v_{c}(f)
$$


for a Riemann-integrable function $f$. Indeed, let us consider the integral

$$
V_{n}(t)=\frac{1}{2 \pi} \int_{0}^{2 \pi} \omega_{n}(t-\tau) f(\tau) d \tau
$$

and its approximating sums

$$
V_{n, m}(t)=\frac{1}{m} \sum_{\nu=0}^{m-1} \omega_{n}\left(t-\frac{2 \pi \nu}{m}\right) f\left(\frac{2 \pi \nu}{m}\right)
$$

Lemma 3 and definition (2) imply

$$
v_{c}\left(V_{n, m}\right) \leqq v_{c}\left(f\left(\begin{array}{c}
2 \pi \nu \\
m
\end{array}\right)\right) \leqq v_{c}(f)
$$

or

$$
v_{c}\left(V_{n, m}\right) \leqq v_{c}(f) .
$$

Since $V_{n, m}(t) \rightarrow V_{n}(t)$ for all $t$, as $m \rightarrow \infty$, the last inequality evidently implies (1.9). An "approximation argument" extending (1.9) to a more comprehensive class of functions is easy, but hardly deserves to be presented here.

1.3. A first proof of Theorem 1. The first inequality (13) is immediate and so the essential assertion of Theorem 1 consists in the inequality

$$
Z_{c}\left(V_{n}\right) \leqq v_{c}(f)
$$

If $v_{c}(f) \geqq 2 n$ there is nothing to prove ; also if $v_{c}(f)=0$ for then $V_{n}(t)$ clearly can not vanish. Let us assume, then, that $f(t)$ is " normalized" according to $\S 1.2$, and that $0<v_{c}(f)=2 k<2 n$, and let us divide the unit circumference into the $2 k$ consecutive arcs (1.7) which satisfy the conditions (1.8) and $\left(1.8^{\prime}\right)$. We may then write the Fourier coefficients of $f(t)$ in the form

$$
\begin{gathered}
a_{\jmath}=\frac{1}{\pi} \int_{0}^{2 \pi} f(t) d t=\sum_{s=1}^{k} \frac{1}{\pi} \int_{I_{2 s-1}}|f(t)| d t-\sum_{s=1}^{k} \frac{1}{\pi} \int_{I_{2 s}}|f(t)| d t \\
a_{\nu}=\frac{1}{\pi} \int_{0}^{2 \pi} f(t) \cos \nu t d t=\sum_{1}^{k} \frac{1}{\pi} \int_{I_{2 s-1}}|f(t)| \cos \nu t d t \\
\quad-\sum_{1}^{k} \frac{1}{\pi} \int_{I_{2 s}}|f(t)| \cos \nu t d t, \\
b_{\nu}=\frac{1}{\pi} \int_{0}^{2 \pi} f(t) \sin \nu t d t=\sum_{1}^{k} \frac{1}{\pi} \int_{I_{2 s-1}}|f(t)| \sin \nu t d t
\end{gathered}
$$




$$
\begin{array}{r}
-\sum_{1}^{k} \frac{1}{\pi} \int_{I_{2 s}}|f(t)| \sin \nu t d t . \\
\nu=1, \cdots, n ;
\end{array}
$$

Consider in the $2 n$-dimensional space $E_{2 n}$ the closed curve $\Gamma$ defined in parametric form by $\cos \nu t, \sin \nu t(\nu=1, \cdots, n ; 0 \leqq t \leqq 2 \pi)$. To the division (1.7) of the circumference into the $\operatorname{arcs} I_{\mu}$, corresponds a division of $\Gamma$ into ares

$$
\Gamma_{1}, \Gamma_{2}, \cdots, \Gamma_{2 k},
$$

where we think of the arc $\Gamma_{\mu}$ as carrying the positive mass

$$
\frac{1}{\pi} \int_{T_{\mu}}|f(t)| d t
$$

This mass has a centroid the coordinates of which, multiplied by (1.13), are

$$
\frac{1}{\pi} \int_{I \mu}|f(t)| \cos \nu t d t, \frac{1}{\pi} \int_{I_{\mu}} \mid f(t) \sin \nu t d t \quad(\nu=1, \cdots, n) .
$$

By a well known theorem of Carathéodory the mass (1.13) of $\Gamma_{\mu}$ may be concentrated in a finite number of points along $\Gamma_{\mu}$ so as to produce the same centroid (1.14). This we do for each of the arcs (1.12). Arranging all these points in cyclic order along $\Gamma$ we obtain points $\tau_{1}, \tau_{2}$, $\cdots, \tau_{m}$ and corresponding coefficients $c_{1}, c_{2}, \cdots, c_{m}$ where $(-1)^{\mu-1} c_{j}>0$ when $\tau$, belongs to $I_{\mu}$. In view of the relations (1.11) we obtain

$$
\begin{gathered}
a_{0}=\sum_{j=1}^{m} c_{j}, a_{\nu}=\sum_{j=1}^{m} c_{j} \cos \nu \tau_{j}, b_{\nu}=\sum_{j=1}^{m} c_{j} \sin \nu \tau_{j}, \\
v_{c}\left(c_{j}\right)=2 k=v_{c}(f) .
\end{gathered}
$$

We consider now the trigonometric polynomial

$$
F(t)=\frac{1}{2} \sum_{j=1}^{m} c_{j} \omega_{n}\left(t-\tau_{j}\right)
$$

and claim that

$$
F(t)=V_{n}(t)
$$

Indeed, by $\left(6^{\prime}\right)$

$$
\begin{aligned}
F(t) & =\frac{1}{2} \sum_{j=1}^{m} c_{j} \omega_{n}\left(t-\tau_{j}\right) \\
& =\sum_{j} c_{j}\left\{\frac{1}{2}+\sum_{\nu=1}^{n} \begin{array}{c}
n ! \\
(n-\nu) !(n+\nu) !
\end{array} \quad \cos \nu\left(t-\tau_{j}\right)\right\}
\end{aligned}
$$




$$
=\sum_{j} c_{j}\left\{\frac{1}{2}+\sum_{\nu=1} \frac{n !}{(n-\nu) !} \frac{n !}{(n+\nu) !}\left(\cos \nu t \cos \nu \tau_{j}+\sin \nu t \sin \nu \tau_{j}\right)\right\},
$$

and interchanging the order of summations, we obtain by (1.15)

$$
F(t)=\frac{1}{2} a_{0}+\sum_{\nu=1}^{n} \frac{n !}{(n-\nu) !} \frac{n !}{(n+\nu) !}\left(a_{\nu} \cos \nu t+b_{\nu} \sin \nu t\right)
$$

which is identical with $V_{n}(t)$ by (12). Finally, by (1.17), (1.18), (1.16) and Lemma 3

$$
Z_{c}\left(V_{n}\right)=Z_{c}(F) \leqq v_{c}\left(c_{j}\right)=v_{c}(f),
$$

which proves the inequality (1.10).

2. A second proof of Theorem 1. The foregoing proof is based on Sylvester's result which we stated as Lemma 1 . We shall now prove Theorem 1 without assuming the knowledge of this result.

We transform (7) by changing the variables. Setting

$$
x=\tan \frac{t}{2}, \quad \xi=-\cot \frac{\tau}{2},
$$

we obtain from (7) (by steps similar to those exhibited following (1.3)) that

$$
\left(1+x^{2}\right)^{n} V_{n}(2 \arctan x)=\int_{-\infty}^{\infty}(x-\xi)^{2 n} \frac{(n !)^{2} 2^{2 n}}{(2 n) ! \pi} \frac{f(-2 \operatorname{arccot} \xi)}{\left(1+\xi^{2}\right)^{n+1}} d \xi
$$

This relation is contained in the more general

$$
P(x)=\int_{-\infty}^{\infty}(x-\xi)^{m} A(\xi) d \xi
$$

where $m$ is a positive integer and the integral $\int_{-\infty}^{\infty} \xi^{m} A(\xi) d \xi$ is absolutely convergent; $P(x)$ is by the structure of the formula (2.2) a polynomial of degree not higher than $m$.

We consider the following quantities connected with (2.2):

$N$ the number of real zeros of $P(x)$, counted with multiplicity;

$v$ the number of variations of sign of $A(\xi)$ in the open interval $-\infty<\xi<\infty$;

$\operatorname{sgn} A(\infty)$ is the constant sign, different from 0 , that $A(\xi)$ possesses whenever it is different from 0 in a suitably chosen interval $\omega<\xi<\infty$; waessume here that $A(\xi)$ is normalized in the sense of $\$ 1.2$; 
$\operatorname{sgn} A(-\infty)$ is similarly defined ;

$$
\eta=\frac{1}{2} \cdot \operatorname{sgn} A(\infty)-\operatorname{sgn}(-1)^{m} A(-\infty) \mid
$$

so that $\eta$ is either 0 or 1 ;

$$
V=v+\eta \text {. }
$$

In fitting (2.1) into the more general pattern (2.2), we can assume without loss of generality (by rotating the circle through an appropriate angle) that $V_{n}(\pi) \neq 0$, that $f(t)$ is normalized in the sense of $\S 1.2$, and that 0 is an interior point of one of the intervals of constant sign considered there, so located that, for some positive $\varepsilon, f(t)$ takes some nonvanishing values in both intervals $-\varepsilon<t<0$ and $0<t<\varepsilon$. Under these circumstances, in the particular case (2.1),

$$
\begin{gathered}
m=2 \mathrm{n}, \\
A(-\infty)=A(\infty), \\
\eta=0, \\
V=v=v_{c}(f), \\
N=Z_{c}\left(V_{n}\right),
\end{gathered}
$$

and so Theorem I is an immediate consequence of the following.

LEMMA $4 . \quad N \leqq V$.

We need several steps to prove Lemma 4.

(a) There are some particular cases in which Lemma 4 is obvious.

If $P(x)$ vanishes identically there is nothing to prove since in this case, by definition, $N=0$.

If $V \geqq m$ there is nothing to prove since, of course, $N \leqq m$.

If $v=0$ and $m$ is even (so that $V=\eta=0$ ) then $P(x)$ will have for all real $x$ the constant sign of $A(\xi)$ and so $N=0$ as it should be according to Lemma 4.

If $v=0$ and $m$ is odd (so that $V=\eta=1$ ) then $m-1$ is even and so

$$
P^{\prime}(x)=\int_{-\infty}^{\infty}(x-\xi)^{m-1} m A(\xi) d \xi
$$

has a constant sign for all $x$, by what we have just said. Therefore, $P(x)$ is monotone and $N=1$ which agrees with Lemma 4 .

And so we may and shall assume in the sequel that

$$
1 \leqq v \leqq V \leqq m-1
$$


(b) Let $c$ be a point of change of sign for $A(\xi)$; that is, $c$ is the common endpoint of two contiguous intervals in each of which $A(\xi)$ keeps a constant sign, yet the two signs (cf. \$1.2) considered are opposite. The number of such points is $v$ and we have assumed (2.3).

We assert that at least one of the $m-1$ quantities $P^{\prime}(c), P^{\prime \prime}(c), \cdots$, $P^{(m-1)}(c)$ is different from 0 . If this assertion were wrong, the integral

$$
\int_{-\infty}^{\infty}(\xi-c)^{\mu} A(\xi) d \xi
$$

would vanish for $\mu=m-1, \cdots, 2,1$ and, as a linear combination of these integrals,

$$
\int_{-\infty}^{\infty}(\xi-c) Q(\xi) A(\xi) d \xi
$$

would vanish for any polynomial $Q(\xi)$ of degree not exceeding $m-2$. Yet this is certainly false if

$$
Q(\xi)=\left(x-c_{1}\right)\left(x-c_{2}\right) \cdots\left(x-c_{v-1}\right)
$$

where $c, c_{1}, c_{2}, \cdots, c_{v-1}$ are all the points of change of sign of $A(\xi)$; observe (2.3) in computing the degree of $Q(\xi)$. In fact, with (2.5) the integrand in (2.4) has a constant sign and so the integral (2.4) cannot vanish.

We have seen by the way, that under the condition (2.3) $P(x)$ cannot vanish identically.

(c) Set

$$
\begin{aligned}
G(x) & =P(x)(x-c)^{-m} \\
P^{*}(x) & =(x-c)^{m+1} G^{\prime}(x) \\
& =(x-c) P^{\prime}(x)-m P(x) \\
& =\int_{-\infty}^{\infty}(x-\xi)^{m-1} A^{*}(\xi) d \xi
\end{aligned}
$$

where

$$
A^{*}(\xi)=m(\xi-c) A(\xi)
$$

and let $N^{*}, m^{*}, v^{*}, \eta^{*}, V^{*}$ be just so connected with $P^{*}(x)$ and $A^{*}(\xi)$ as $N, m, v, \eta$ and $V$ are with $P(x)$ and $A(\xi)$. Obviously

$$
\begin{aligned}
m^{*} & =m-1 \\
v^{*} & =v-1 \\
\operatorname{sgn} A^{*}(\infty) & =\operatorname{sgn} A(\infty) \\
\operatorname{sgn} A^{*}(-\infty) & =-\operatorname{sgn} A(-\infty)
\end{aligned}
$$

and so $\eta^{*}=\eta$. Combining this with (2.9), we obtain 


$$
V^{*}=V-1
$$

We intend to prove Lemma 4 by mathematical induction with respect to $V$. In fact, we have already proved Lemma 4 in the particular case $V=0$ under (a). We therefore assume $V \geqq 1$, cf. (2.3), and that Lemma 4 has been proved for the preceding value (2.10), and so we take for granted that

$$
N^{*} \leqq V^{*}
$$

(d) Let $k$ denote the number of those zeros of $P(x)$ that coincide with the point $c$; obviously $k \geqq 0$, and, by (b),

$$
k \leqq m-1 \text {. }
$$

Let $k^{*}$ denote the number of those zeros of $P^{*}(x)$ that coincide with $c$. We set

$$
N=k+l, \quad N^{*}=k^{*}+l^{*} .
$$

The quantities $l$ and $l^{*}$, defined by (2.13), enumerate those zeros of $P(x)$ and $P^{*}(x)$, respectively, that fall into one or the other of the two open intervals $-\infty<x<c$ and $c<x<\infty$.

We note the critical term of the expansion of $P(x)$ around the point $x=c$,

$$
P(x)=\frac{P^{(k)}(c)(x-c)^{k}}{k !}+\cdots, P^{(k)}(c) \neq 0
$$

By (2.6) and (2.12), $G(x)$ has a pole at the point $c$ and (2.7) yields

$$
P^{*}(x)=\frac{(k-m) P^{(k)}(c)(x-c)^{k}}{k !}+\cdots
$$

We infer that $P^{*}(x)$ has just as many zeros at the point $c$ as $P(x)$ :

$$
k^{*}=k \text {. }
$$

By the way, we have seen that $P^{*}(x)$ does not vanish identically.

(e) It remains to consider the real zeros different from $c ; P(x)$ or, which is the same, $G(x)$ has $l$ such zeros, and $P^{*}(x)$ or, which is the same, $G^{\prime}(x)$ has $l^{*}$ such zeros. These zeros are distributed somehow in the two open intervals, $-\infty<x<c$ and $c<x<\infty$.

By the theorem of Rolle, in each of these intervals at most one zero can be lost in the passage from $G(x)$ to $G^{\prime}(x)$, so that 


$$
l^{*} \geqq l-2 ;
$$

this information is correct, but insufficient for our purpose. We shall obtain, however, additional information by using the following remark (cf. [9, vol. 2, p. 39, problem 14]).

No zero can be lost in the xassage from $G(x)$ to $G^{\prime}(x)$ in the interval $(-\infty, c)$ if

$$
\operatorname{sgn} G(-\infty)=\operatorname{sgn} G^{\prime}(-\infty)
$$

and no zero can be lost in this passage in the interval $(c, \infty)$ if

$$
\operatorname{sgn} G(\infty)=-\operatorname{sgn} G^{\prime}(\infty) .
$$

The signs mentioned in (2.16) and (2.17) refer to a certain neighborhood of $-\infty$ or $\infty$ and, as $G(x)$ has only a finite number of zeros, they are certainly different from 0 .

(f) We know, cf. (b), that the polynomial $P(x)$ does not vanish identically. We set

$$
P(x)=b_{0} x^{m}+b_{1} x^{m-1}+\cdots+b_{m}
$$

and distinguish two cases.

Case I. If $b_{0}=0$, there is an $s$ such that $b_{0}=b_{1}=\cdots=b_{s-1}=0, b_{s} \neq 0$ and so we easily find the initial terms in the expansions around $\infty$ :

$$
G(x)=\frac{b_{s}}{x^{s}}+\cdots, \quad \frac{G^{\prime}(x)}{G(x)}=-\frac{s}{x}+\cdots
$$

In this case, both conditions (2.16) and (2.17) are satisfied, and, by the final remark under (e), we can improve (2.15) to

$$
l^{*} \geqq l .
$$

Case II. Now

$$
b_{\mathrm{v}}=\int_{-\infty}^{\infty} A(\xi) d \xi \neq 0
$$

and the expansions around $\infty$ begin

$$
\begin{aligned}
& G(x)=b_{0}+\frac{m c b_{0}+b_{1}}{x}+\cdots \\
& G^{\prime}(x)=-\frac{m c b_{0}+b_{1}}{x^{2}}+\cdots
\end{aligned}
$$

where 


$$
m c b_{0}+b_{1}=m \int_{-\infty}^{\infty} A(\xi)(c-\xi) d \xi .
$$

We again distinguish two cases.

Subcase II, 1. If $v=1, c$ is the only point of change of sign of $A(\xi)$, the integrand in (2.23) is of constant sign, and so the integral is different from 0 .

Subcase II, 2. If $v \geqq 2$, the integral (2.23) could vanish. Yet in this case $A(\xi)$ has at least another point of change of sign, $c_{1}$, and we say that (2.23) and

$$
m \int_{-\infty}^{\infty} A(\xi)\left(c_{1}-\xi\right) d \xi
$$

cannot vanish simultaneously: in fact, their difference is

$$
m\left(c_{1}-c\right) \int_{-\infty}^{\infty} A(\xi) d \xi=m\left(c_{1}-c\right) b_{0} \neq 0
$$

by our present assumption (2.20). Therefore, assuming that the point of change was properly selected from the start (which boils down to a proper choice of notation) we may assume that (2.23) is different from 0 , also in the present subcase.

Finally, in both subcases, we conclude from (2.21) and (2.22)

$$
\lim _{x \rightarrow \pm \infty} \frac{x^{2} G^{\prime}(x)}{G(x)}=-\frac{m c b_{0}+b_{1}}{b_{0}} \neq 0
$$

and we see that just one of the two conditions (2.16) and (2.17) is fulfilled. Therefore, by the final remark under (e), we can improve (2.15) to

$$
l^{*} \geqq l-1
$$

Thus, even in the less favorable of the two cases I and II, we have (2.24). Combining this with (2.13) and (2.14), we obtain

$$
N^{*} \geqq N-1
$$

and hence and from (2.10) and (2.11) we obtain

$$
V-1=V^{*} \geqq N^{*} \geqq N-1
$$

or $V \geqq N$, which is the desired conclusion of Lemma 4 .

The foregoing somewhat involved proof becomes more understandable if it is compared with the proof for Lemma 1 given in [7] or in [9, vol. 2, p. 50, problem 79]. 


\section{PART II. SOME APPLICATIONS OF THE VARIATION DIMINISHING PROPERTY OF $V$-MEANS}

3. A theorem of Ch. Sturm and A. Hurwitz. Let $f(t)$ be a realvalued, integrable, periodic function of period $2 \pi$. Let

$$
f(t)=\frac{1}{2} a_{\nu}+\sum_{\nu=1}^{\infty}\left(a_{\nu} \cos \nu t+b, \sin \nu t\right)
$$

be its Fourier expansion. Suppose that the partial sum

$$
S_{n}(t)=\frac{1}{2} a_{0}+\sum_{\nu=1}^{n}\left(a_{\nu} \cos \nu t+b_{\nu} \sin \nu t\right)
$$

is known. What can we say about the number $v_{c}(f)$ of changes of sign of $f(t)$ in a period? An answer is immediate: Knowing (3.2), we can compute (11), the $n$th $V$-mean of $f(t)$, and we must have

$$
v_{c}(f) \geqq Z_{c}\left(V_{n}\right)
$$

by Theorem 1 .

The information provided by this inequality is strongest when the right hand side attains its largest value $2 n$. There is a simple sufficient condition for this eventuality which we record as follows.

COROLLARY 1. If

$$
\begin{aligned}
& \left(a_{n}^{2}+b_{n}^{2}\right)^{1 / 2}>\left(\begin{array}{c}
2 n \\
1
\end{array}\right)\left(a_{n-1}^{2}+b_{n-1}^{2}\right)^{1 / 2}+\left(\begin{array}{c}
2 n \\
2
\end{array}\right)\left(a_{n-2}^{2}+b_{n-2}^{2}\right)^{1 / 2}+\cdots \\
& +\left(\begin{array}{c}
2 n \\
n-1
\end{array}\right)\left(a_{1}^{2}+b_{1}^{2}\right)^{1 / 2}+\frac{1}{2}\left(\begin{array}{c}
2 n \\
n
\end{array}\right)\left|a_{v}\right|,
\end{aligned}
$$

then every function $f(t)$ having (3.2) as the nth partial sum of its Fourier series, must change sign within a period at least $2 n$ times.

Indeed, it is clear by (3.4) that the last term of the expression (11) for $V_{n}(t)$ so predominates that $V_{n}(t)$ has $2 n$ simple zeros, hence $Z_{c}\left(V_{n}\right)=2 n$. The statement now follows from (3.3).

We obtain a classical result [2, pp. 572-574] as a very special case.

CoRollary 2. If $a_{0}=a_{1}=b_{1}=\cdots=a_{n-1}=b_{n-1}=0, a_{n}^{2}+b_{n}^{2}>0$, then $v_{c}(f) \geqq 2 n$.

The following is an equivalent formulation. If $a_{n}^{2}+b_{n}^{2}>0$ then

$$
v_{c}\left(f(t)-S_{n-1}(t)\right) \geqq 2 n .
$$

This second formulation is especially interesting and intuitive because it shows that the graph of the partial sum $S_{n-1}(t)$ must cross the graph 
of $f(t)$ at least $2 n$ times. Hurwitz's proof of Corollary 2 is direct and elementary. However, his classical argument is no longer available to establish other special cases such as the following.

If

$$
f(t)=\frac{1}{2}+\sum_{\nu=1}^{n} \cos \nu t+\sum_{\nu=n+1}^{\infty}\left(a_{\nu} \cos \nu t+b_{\nu} \sin \nu t\right)
$$

then

$$
v_{c}(f) \geqq 2 n
$$

For in this case $V_{n}(t)=\frac{1}{2} \omega_{n}(t)$, hence $Z_{c}\left(V_{n}\right)=2 n$ so that (3.3) implies the result. Such particular examples are easily constructed and we see no other way of proving them except by the fundamental inequality (3.3).

4. The simplest Pólya-Wiener result concerning high order derivatives of periodic functions. Let $f(t)$ be a real function of period $2 \pi$ which is infinitely often differentiable. Let us consider its zeros and also the zeros of its successive derivatives. Counting multiplicities as usual we set

$$
N^{(k)}=Z_{c}\left(f^{(k)}\right), \quad k=0,1, \cdots,
$$

and assume all these numbers to be finite. A familiar application of Rolle's theorem shows that

$$
N^{(0)} \leqq N^{(1)} \leqq \cdots \leqq N^{(k)} \leqq N^{(k+1)} \leqq \cdots .
$$

Can this sequence remain bounded? This is surely the case if $f(t)$ is a trigonometric polynomial. The truth of the converse is stated by the following proposition due to Pólya and Wiener [8.]

CoRollary 3. If the sequence (4.1) is bounded and

$$
\lim N^{(k)}=2 m,
$$

then $f(t)$ is a trigonometric polynomial of exact order $m$.

Indeed, let (3.1) be the Fourier series of $f(t)$. It is known to converge under our assumptions and the expansion of $f^{(k)}(t)$ is obtained by formal differentiations of the expansion of $f(t)$. Let us assume that for a certain $n$

$$
a_{n}^{2}+b_{n}^{2}>0 \text {. }
$$

It is clear then from the form of the Fourier series for $f^{(k)}(t)$ that this series will satisfy the inequality (3.4) of Corollary 1, provided only that 
$k$ is sufficiently large, $k>K$ say. But then by Corollary 1

$$
N^{(k)} \geqq v_{c}\left(f^{(k)}\right) \geqq 2 n, \quad(\text { if } k>K) .
$$

Thus (4.2) and (4.3) imply that $n \leqq m$, and $f(t)$ must reduce to a trigonometric polynomial of order $q \leqq m$. On the other hand, if $f(t)$ is such a polynomial, $N^{(k)} \leqq 2 q$ which implies $2 m \leqq 2 q$ or $m \leqq q$, hence $q=m$ and the theorem is established.

5. The graphic behavior of $\boldsymbol{V}$-means. We now wish to discuss the shape-preserving properties of the $V$-means which are implicitly contained in the fundamental inequality

$$
Z_{c}\left(V_{n}\right) \leqq v_{c}(f) .
$$

It shows that $V_{n}(t)$ can't oscillate about zero more frequently than $f(t)$ does. But there is nothing peculiar about the level zero. Indeed, if $r$ is any real, then $f(t)=\gamma$ implies $V_{n}(t)=\gamma$. Thus we may replace in (5.1) $f$ and $V_{n}$ by $f-\gamma$ and $V_{n}-\gamma$, respectively, obtaining the inequality

$$
Z_{c}\left(V_{n}-\gamma\right) \leqq v_{c}(f-\gamma) \text {. }
$$

A second remark is based on the obvious known fact (see [5, p. 191]) that if $f(t)$ is absolutely continuous then $V_{n}^{\prime}(t)$ is the $V$-mean of $f^{\prime}(t)$. But then (5.1) immediately gives

$$
Z_{c}\left(V_{n}^{\prime}\right) \leqq v_{c}\left(f^{\prime}\right)
$$

This operation may naturally be repeated giving

$$
Z_{c}\left(V_{n}^{(k)}\right) \leqq v_{c}\left(f^{(k)}\right),
$$

which is valid depending on how many derivatives $f(t)$ possesses. For instance, if

$$
f(t) \in C^{\prime \prime}
$$

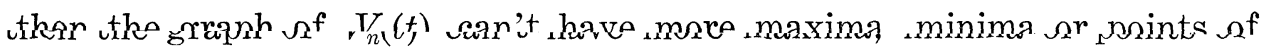
inflexion than the corresponding numbers for the graph of $f(t)$.

It is desirable, however, to discuss this phenomenon for functions of a lower degree of smoothness and the following developments aim to do that. We consider the class $D_{0}$ of real periodic functions $f(t)$, of bounded variation, normalized by $2 f(t)=f(t+0)+f(t-0)$. A subclass of $D_{0}$ is the class $D_{1}$ of functions satisfying the classical Dirichlet conditions. By $f(t) \in D_{1}$ we mean that the circle can be dissected into a finite number of consecutive open arcs in each of which $f(t)$ is monotone in the wide sense.

With each $f(t) \in D_{1}$ we associate an even non-negative integer $S(f)$, 
called the number of sense-reversals of $f(t)$ and defined as follows. Consider, for a given natural number $k$, the periodic sequence of ordinates

$$
f_{\nu}=f\left(\frac{2 \pi \nu}{k}\right)=f(\nu h), \quad h=2 \pi / k,
$$

of period $k$, and the likewise periodic sequence of differences

$$
\Delta f_{\nu}=f\left(\frac{2 \pi(\nu+1)}{k}\right)-f\left(\frac{2 \pi \nu}{k}\right),
$$

$\nu=0,1,2, \cdots, n-1$. We now define $S(f)$ by

$$
S(f)=\lim _{k \rightarrow \infty} v_{c}(\Delta f \nu)=\max _{k} v_{c}\left(\Delta f_{\nu}\right) .
$$

The reader is urged to supply proof for the statements implied in this definition; it depends on an analysis of the finitely many points which have no neighborhood in which $f(t)$ is monotone. If in addition to $f(t) \in D_{1}$ we assume that $f(t) \in C^{\prime}$ then evidently $S(f)=v_{c}\left(f^{\prime}\right)$.

Our substitute for (5.3) for the class $D_{1}$ is given by the following.

Theorem 3. If $f(t) \in D_{1}$ then

$$
v_{c}\left(V_{n}^{\prime}\right)=S\left(V_{n}\right) \leqq S(f) .
$$

The proof is very simple. Besides the $V$-mean

$$
V_{n}(t)=\frac{1}{2 \pi} \int_{0}^{2 \pi} \omega_{n}(t-\tau) f(\tau) d \tau
$$

we consider the approximating sums

$$
V_{n, k}(t)=\frac{1}{k} \sum_{\nu} \omega_{n}(t-\nu h) f_{\nu},
$$

Replacing $t$ by $t+h$ we obtain

$$
V_{n, k}(t+h)=\frac{1}{k} \sum_{\nu} \omega_{n}(t-\nu h) f_{\nu+1}
$$

and therefore

$$
\Delta V_{n, k} / h=\left(V_{n . k}(t+h)-V_{n, k}(t)\right) / h=\frac{1}{2 \pi} \sum_{\nu} \omega_{n}(t-\nu h) \Delta f_{\nu} .
$$

By Lemma 3 , in view of (5.6), we obtain $v_{c}\left(\Delta V_{n, k} / h\right) \leqq v_{c}(\Delta f) \leqq S(f)$ or

$$
v_{c}\left(\Delta V_{n, k} / h\right) \leqq S(f) \text {. }
$$


Because the difference quotient (5.8) converges to $V_{n}^{\prime}(t)$, as $k \rightarrow \infty$, for all $t$, the last inequality implies (5.7).

There is a similar significant substitute for (5.4) if $k=2$. In order to formulate it we define a class of functions $f(t)$ which we denote by $D_{2}$ : By $f(t) \in D_{2}$ we mean that the circle can be dissected into a finite number of consecutive open ares in each of which $f(t)$ is continuous and convex, or concave, or linear. It is clear that $D_{2} \subset D_{1}$.

With each $f(t) \in D_{2}$ we associate an even non-negative integer $T(f)$, called the number of turn-reversals of $f(t)$ and defined as follows: Besides the $\Delta f \nu$ we consider the periodic sequence of second differences

$$
\delta^{2} f_{\nu}=f_{\nu+1}-2 f_{\nu}+f_{\nu-1}
$$

and define $T(f)$ by

$$
T(f)=\lim _{k-\infty} v_{c}\left(\delta^{2} f_{\nu}\right)=\sup _{k} v_{c}\left(\delta^{2} f_{\nu}\right) .
$$

Again a proof of the equality of the last two expressions requires the consideration of the points (finite in number) which have no neighborhood in which $f(t)$ is convex, or concave. If in addition to $f(t) \in D_{2}$ we assume that $f(t) \in C^{\prime \prime}$ then evidently

$$
T(f)=v_{c}\left(f^{\prime \prime}\right) \text {. }
$$

A substitute of (5.4) for $k=2$ is given by

THEOREM 4. If $f(t) \in D_{2}$ then

$$
v_{c}\left(V_{n}^{\prime \prime}\right)=T\left(V_{n}\right) \leqq T(f) .
$$

The proof is so very similar to the proof of Theorem 3 that it suffices to indicate the main points. In place of (5.8) we now start from the second order difference quotient

$$
\begin{aligned}
\delta^{2} V_{n, k} / h^{2} & =\left(V_{n, k}(t+h)-2 V_{n, k}(t)+V_{n, k}(t-h)\right) / h^{2} \\
& =\frac{1}{2 \pi h} \sum_{\nu} \omega_{n}(t-\nu h) \delta^{2} f_{\nu},
\end{aligned}
$$

and observe that on the one hand it converges to $V_{n}^{\prime \prime}(t)$, on the other hand by Lemma 3 and (5.9)

$$
v_{c}\left(\delta^{2} V_{n, k} / h^{2}\right) \leqq T(f) .
$$

This last inequality implies (5.10) on letting $k \rightarrow \infty$.

The following remarks concerning the simplest elements of $D_{1}$ and $D_{2}$ are called for: 1 . If $f(t)=$ const. then clearly $S(f)=0$ and $T(f)=0$. Conversely, either of these relations is easily seen to imply that $f(t)=$ const. 2. The first non-trivial case is 


$$
S(f)=2 \text {. }
$$

Functions $f(t)$ satisfying (5.11) are in a way the simplest non-constant periodic functions and may aptly be called periodically monotone. Likewise functions with

$$
T(f)=2
$$

may be called periodically convex.

It is easily shown that (5.12) implies (5.11). That these new terms are appropriate is also shown by the following two statements.

1. If the periodic function $f(t)$ is monotone (non-constant) in $-\pi<t<\pi$ then $S(f)=2$, that is, $f(t)$ is periodically monotone.

2. If the periodic function $f(t)$ is convex or concave (non-constant) in $-\pi<t<\pi$ then $T(f)=2$, that is $f(t)$ is periodically convex.

Observe that the distinction between "increasing" and "decreasing" as well as between "convex" and "concave", drops out for periodic functions.

We conclude our short excursus into "descriptive function theory" with a few examples:

$$
\begin{aligned}
S(\sin t) & =T(\sin t)=2 . \\
S(|\sin t|) & =T(|\sin t|)=4 .
\end{aligned}
$$

If $f(t)=\sin t+1$ in $(-\pi, 0)$ and $f(t)=\sin t$ in $(0, \pi)$ then

$$
S(f)=T(f)=6 .
$$

If $f(t)=\sin t+t$ in $0<t<2 \pi$, then

$$
S(f)=2, T(f)=4 \text {. }
$$

From these examples we see that

$$
S(f) \leqq T(f)
$$

and this inequality is generally true. We see this if we observe that for a periodic sequence (5.5) we always have

$$
v_{c}\left(f_{\nu}\right) \leqq v_{c}\left(\Delta f_{\nu}\right) \leqq v_{c}\left(\delta^{2} f_{\nu}\right)
$$

In view of (5.6), (5.9) and the corresponding relations

$$
v_{c}(f)=\lim _{k \rightarrow \infty} v_{c}\left(f_{\nu}\right)=\max _{k} v_{c}\left(f_{\nu}\right),
$$

we conclude that

$$
v_{c}(f) \leqq S(f) \leqq T(f)
$$


It is of some interest to show that the remarkable properties of the third Cesàro means established by L. Fejér in his Theorems 1,2 and $3[1$, p. 82 and p. 86] are also enjoyed by the de la Vallée Poussin means $V_{n}(t)$. Thus Fejér's work suggests the following

THEOREM 5. If $f(t)$ is an odd periodic function which is positive and concave in the range $0<t<\pi$, then

$$
0<V_{n}(t) \leqq f(t) \text { if } 0<t<\pi(n \geqq 1) .
$$

Moreover, the function $V_{n}(t)$ is also concave in $0<t<\pi$.

The last statement and the first inequality (5.15) are easily proved. Indeed, it is clear that

$$
v_{c}(f)=S(f)=T(f)=2 .
$$

Observe also that $V_{n}(t) \not \equiv 0$ if $n \geqq 1$, for $V_{n}(t) \equiv 0$ would imply $S_{n}(t) \equiv 0$, hence also $v_{c}(f) \geqq 2 n+2 \geqq 4$ (by Corollary 2) which contradicts (5.16). By Theorem 1 and (5.16) surely

$$
Z_{c}\left(V_{n}\right)=2 .
$$

Since $V_{n}(t)$ is a sine polynomial it vanishes at 0 and $\pi$. By (5.17) these zeros are simple and the only zeros of $V_{n}(t)$. Also by (5.16) and Theorems 3 and 4 we conclude that

$$
v_{c}\left(V_{n}^{\prime}\right)=v_{c}\left(V_{n}^{\prime \prime}\right)=2 .
$$

These remarks show that $V_{n}(t)$ or perhaps $-V_{n}(t)$ enjoy the properties to be established. That $V_{n}(t)$, rather than $-V_{n}(t)$, has these properties is shown by observing that

$$
V_{n}^{\prime}(0)=c \int_{-\pi}^{\pi}\left(\cos \frac{\tau}{2}\right)^{2 n-1} \sin \frac{\tau}{2} f(\tau) d \tau, \quad c>0,
$$

(obtained from (7) by differentiation) has a positive integrand and is therefore positive.

To establish the second inequality (5.15) or

$$
V_{n}(t) \leqq f(t)
$$$$
0<t<\pi \text {, }
$$

is a little more troublesome and we resort to Fejér's own method. We consider the "roof-function"

$$
\hat{f}(t)=\left\{\begin{array}{ll}
\frac{b}{a} t & \text { if } 0 \leqq t \leqq a \\
b \frac{\pi-t}{\pi-a} & \text { if } a \leqq t \leqq \pi
\end{array} \quad 0<a<\pi, b>0,\right.
$$


and denote again by $\hat{f}(t)$ its odd periodic extension. We now observe that indeed

$$
V_{n}(t)<\hat{f}(t),
$$$$
0<t<\pi,
$$

for these special functions. Since we already know from our previous discussion that $\hat{V}_{n}(t)$ is positive and concave in $(0, \pi)$, the inequality (5.20) is perfectly clear as soon as we can prove that

$$
\hat{V}_{n}^{\prime}(0)<\hat{f}^{\prime}(0), \quad \hat{V}^{\prime}(\pi)>\hat{f}^{\prime}(\pi),
$$

These inequalities, however, follow immediately from previous remarks. Since $\hat{f}(t)$ is continuous, $\hat{V}_{n}^{\prime}(t)$ is the $V$-mean of $\hat{f}^{\prime}(t)$. Since $\hat{f}^{\prime}(0)=$ sup $\hat{f}^{\prime}(t), \hat{f}^{\prime}(\pi)=\inf \hat{f}^{\prime}(t)$, we conclude, for instance from (5.2), that

$$
\hat{f}^{\prime}(\pi)<\hat{V}_{n}^{\prime}(t)<\hat{f}^{\prime}(0) \text { for all } t .
$$

The proof of the general inequality (5.18) now follows from the observation that the function $f(t)$ of Theorem 5 may be approximated by appropriate linear combinations of roof-functions with positive coefficients.

6. Convex, and star-shaped, conformal maps of the circle. The following introductory remark (previously made by one of us; see [10, pp. 226-227]) applies to any variation diminishing kernel $\Omega(t)$ as defined by the relations (3), (4) and (5) of our Introduction.

Let

$$
f(t)=f_{1}(t)+i f_{2}(t) \quad\left(f_{1}, f_{2} \text { real-valued }\right)
$$

be a complex-valued continuous function of period $2 \pi$ and let

$$
g(t)=\frac{1}{2 \pi} \int_{0}^{2 \pi} \Omega(t-\tau) f(\tau) d \tau
$$

be its transform; $g(t)$ is evidently also complex-valued periodic and we may write

$$
g(t)=g_{1}(t)+i g_{2}(t), \quad\left(g_{1}, g_{2} \text { real-valued }\right) .
$$

Since $\Omega$ is real and (3) holds it follows that the transforms of $f_{1}(t)$, $f_{2}(t)$ and 1 are $g_{1}(t), g_{2}(t)$ and 1 , respectively. If $A, B, C$ are arbitrary real constants it follows that $A g_{1}(t)+B g_{2}(t)+C$ is the transform of $A f_{1}(t)+B f_{2}(t)+C$. Since $\Omega(t)$ is assumed to be a variation diminishing kernel, we conclude by (5) that the inequality

$$
v_{c}\left(A g_{1}(t)+B g_{2}(t)+C\right) \leqq v_{c}\left(A f_{1}(t)+B f_{2}(t)+C\right)
$$


always holds.

The inequality (6.4) admits a remarkable geometric interpretation. Indeed, let us denote by $\{f\}$ the closed curve traced out by $f(t)$ in the complex plane of the variable $z=x+i y$ as $t$ varies in the range $[0,2 \pi]$, and let $\{g\}$ be the corresponding curve described by $g(t)$. Let the following statement, too simple to be called a theorem, be referred to as a

PRINCIPLE. The curve $\{g\}$ never crosses a straight line more often than the curve $\{f\}$ does.

For if $A x+B y+C=0$ is the equation of a line $L$ then the two members of the inequality (6.4) are identical with the total numbers of crossings of $L$ by $\{g\}$ and $\{f\}$, respectively. In particular we have the

CoRollary 4. If the curve $\{f\}$ is convex then $\{g\}$ is interior to $\{f\}$ and $\{g\}$ is also convex.

Indeed, $\{f\}$ being convex, it crosses any $L$ at most twice, hence also $\{g\}$ crosses any $L$ at most twice and is therefore convex. That $\{g\}$ has no points outside of $\{f\}$ follows already from the properties

$$
\Omega(t) \geqq 0, \frac{1}{2 \pi} \int_{0}^{2 \pi} \Omega(t) d t=1,
$$

and in no way requires the sophisticated condition that $\Omega(t)$ be variation diminishing. On the other hand the conditions (6.5) are by themselves insufficient to enforce the convexity of $\{g\}$. It is also true, however, that the variation diminishing property of $\Omega(t)$ is sufficient but far from necessary for $\{g\}$ to be convex. As an example we mention the periodic kernel

$$
\Omega(t)= \begin{cases}\pi / h & \text { if }-h \leqq t \leqq h \\ 0 & \text { if }-\pi \leqq t \leqq h \text { or } h \leqq t \leqq \pi \quad(0<h<\pi),\end{cases}
$$

which is readily shown to have the "convexity preserving" property of Corollary 4. However, (6.6) is not variation diminishing because it is not periodic totally positive (see [4]).

We now turn to an application of these remarks to conformal maps of the circle, in particular to a proof of Theorem 2 of the Introduction.

Let

$$
F(z)=z+c_{2} z^{2}+c_{3} z^{3}+\cdots
$$

be regular in the unit circle. For a fixed value of $r$ we consider the complex-valued periodic function

$$
f(t ; r)=F\left(r e^{i t}\right)=r e^{i t}+c_{2} r^{2} e^{2 i t}+\cdots, \quad 0 \leqq r \leqq 1 .
$$


By (6.8) and (9) its $V$-means are

$$
\begin{aligned}
V_{n}(t ; r) & =\frac{1}{2 \pi} \int_{0}^{2 \pi} \omega_{n}(t-\tau) F\left(r e^{i r}\right) d \tau \\
& =\frac{1}{\left(\begin{array}{c}
2 n \\
n
\end{array}\right)}\left\{\left(\begin{array}{c}
2 n \\
n+1
\end{array}\right) r e^{i t}+\left(\begin{array}{c}
2 n \\
n+2
\end{array}\right) c_{2} r^{2} e^{2 i t}+\cdots+c_{n} r^{n} e^{n i t}\right\}
\end{aligned}
$$

or

$$
V_{n}(t ; r)=\boldsymbol{V}_{n}\left(r e^{i t}\right),
$$

where $\boldsymbol{V}_{n}(z)$ are the de la Vallée Poussin means of the power series as defined by (15), with $c_{1}=1$. We also record the more explicit expression

$$
\begin{aligned}
V_{n}(z)= & \frac{n}{n+1} z+\frac{n(n-1)}{(n+1)(n+2)} c_{2} z^{2}+\cdots \\
& +\frac{n(n-1) \cdots 1}{(n+1)(n+2) \cdots(2 n)} c_{n} z^{n} .
\end{aligned}
$$

Our Theorem 2 seems now almost self-evident. Indeed, if $F(z) \in K$ then the curve $\left\{\boldsymbol{V}_{n}\left(r e^{i t}\right)\right\}$ is convex by (6.8), (6.9) and Corollary 4. This being true for every $r<1$, we conclude that $\boldsymbol{V}_{n}(z) \in K$. Conversely, if $\boldsymbol{V}_{n}(z) \in K$ for every $n$, then $\left\{\boldsymbol{V}_{n}\left(r e^{i t}\right)\right\}$ is a convex curve for all $n$ and all $r<1$. From the relation

$$
\lim _{n \rightarrow \infty} \boldsymbol{V}_{n}\left(r e^{i t}\right)=F\left(r e^{i t}\right)
$$

we conclude that also $\left\{F\left(r e^{i t}\right)\right\}$ is convex. Hence $F(z) \in K$.

REMARK 1. In order to conclude from (17) that $F(z) \in K$ it is not necessary to assume that the power series (6.7) converges in the unit circle or that it converges at all. Rather the converse part of Theorem 2 holds for a formal power series (6.7). For it is known (see e.g. [9, vol. II, p. 29]) that the assumptions (17) imply that all coefficients of the polynomial (6.10) are bounded in absolute value by $n /(n+1)$. Letting $n \rightarrow \infty$ we obtain $\left|c_{\nu}\right| \leqq 1 \quad(\nu=1,2, \cdots)$ which clearly imply the convergence of (6.7) within the unit circle.

REMARK 2. Let $F(z) \in K$ and hence $V_{n}(z) \in K$. Let $D$ and $D_{n}$ denote the convex domains into which the unit circle is mapped by $F(z)$ and $\boldsymbol{V}_{n}(z)$, respectively. We know by Corollary 4 that

$$
D_{n} \subset \mathrm{D} \text {. }
$$

At this point it is natural to suspect that more is true, namely 
that all the inclusions

$$
D_{1} \subset D_{2} \subset \cdots \subset D_{n} \subset D_{n+1} \subset \cdots
$$

are valid, but we are unable to prove or disprove this.

REMARK 3. Since numerous elements of the class $K$ are explicitly known, Theorem 2 is a ready source of polynomials belonging to $K$. Thus

$$
F^{\prime}(z)=\frac{z}{1-z}=z+z^{2}+\cdots
$$

is in $K$ because it maps the unit circle onto the half-plane $\mathfrak{N}_{z}>-\frac{1}{2}$. The corresponding $V$-means

$$
\boldsymbol{V}_{n}(z)=\frac{1}{\left(\begin{array}{c}
2 n \\
n
\end{array}\right)}\left\{\left(\begin{array}{c}
2 n \\
n+1
\end{array}\right) z+\left(\begin{array}{c}
2 n \\
n+2
\end{array}\right) z^{2}+\cdots+z^{n}\right\}
$$

are a remarkable sequence of polynomials some extremal properties of which might be discussed on another occasion. Of course (6.11) holds. Here the convex boundary of $D_{n}$ touches the line $\mathfrak{N}_{z}=-\frac{1}{2}$ to an order of contact which increases with $n$. Also the inclusions (6.12) can be verified in this special case.

REMARK 4. Observe that the image $D_{1}$ of the unit circle by $V_{1}(z)=\frac{1}{2} z$ is the circle

$$
D_{1}:|z|<\frac{1}{2}
$$

By (6.11) we have $D_{1} \subset D$ for every $F(z) \in K$. This proves the following proposition: The circle (6.15) is covered by every convex map $D$ and (6.15) is the largest circle with this property. That $D_{1}$ is the largest circle is shown by the special function (6.13). This theorem is due to Study, [11, p. 116], and our proof is really identical with Nehari's proof in [6, pp. 223-224].

Remark 5. A comparison of Theorem 2 with Fejér's Theorem IV [1, p. 87] again shows the extent to which the de la Vallée Poussin means of a power series are superior to its third Cesàro means as far as shape-preserving properties are concerned. 
REMARK 6. In $\S 3$ we have seen that from a knowledge of the section (3.2) of the Fourier series (3.1) of $f(t)$ we can infer the information (3.3) concerning the zeros of $f(t)$. Is there a similar result for power series? Specifically, let

$$
F(z)=\sum_{0}^{\infty} c_{2} z^{\nu} \quad\left(c_{0}=1\right)
$$

converge for $|z|<1$ and let, for a certain value of $n$,

$$
\boldsymbol{V}_{n}(z)=\frac{1}{\left(\begin{array}{c}
2 n \\
n
\end{array}\right)} \sum_{\nu=0}^{n}\left(\begin{array}{c}
2 n \\
n+\nu
\end{array}\right) c_{\nu} z
$$

be given and known to have a certain number of zeros within the unit circle. Can we then draw any positive conclusion concerning the existence of zeros of $f(z)$ in the unit circle?

That the answer is negative is very simply shown as follows. With the given $c_{0}=1, c_{1}, \cdots, c_{n}$ derive the expansion

$$
\log \left(1+c_{1} z+\cdots+c_{n} z^{n}\right)=b_{1} z+\cdots+b_{n} z^{n}+\cdots .
$$

But then

$$
F(z)=e^{b_{1} z+\cdots+b_{n} z^{n}}=1+c_{1} z+\cdots+c_{n} z^{n}+\cdots
$$

is a zero-free entire function whose $n$th $\boldsymbol{V}$-mean is precisely the given $\boldsymbol{V}_{n}(z)$.

In concluding this section we wish to point out similar applications concerning the class $\Sigma$ of power series $\sum_{1}^{\infty} b_{2} z^{\nu}$ which map the unit circle onto a univalent domain which is star-shaped with respect to the origin. It is well known that the two classes $K$ and $\Sigma$ are related as follows:

LEMMA 5. $\sum_{1}^{\infty} a_{2} z^{2} \in \Sigma$ if and only if

$$
\sum_{1}^{\infty} \frac{a_{\nu}}{\nu} z^{\nu} \in K
$$

But then Theorem 2 easily implies the following.

COROllary 5. For $F(z) \in \Sigma$ it is necessary and sufficient that $V_{n}(z) \in \Sigma$ for $n=1,2, \cdots$.

\section{APPENDIX I. THE BERNSTEIN POLYNOMIALS}

7. The Bernstein construction is variation diminishing. The purpose of the present appendix is to furnish for functions $f(x)$ defined in a 
finite interval a theory analogous to that given in Parts I and II for periodic functions. It is remarkable that such a theory is provided by the classical Bernstein polynomials. Indeed, let $f(x)$ be defined in $[0,1]$ and let

$$
B_{n}(x)=\sum_{0}^{n} f\left(\frac{\nu}{n}\right)\left(\begin{array}{l}
n \\
\nu
\end{array}\right) x^{\nu}(1-x)^{n-\nu}, \quad n \geqq 1,
$$

be the corresponding Bernstein polynomial (see [5]). Let $Z\left(B_{n}\right)$ denote the number of zeros of $B_{n}(x)$ in the open range $(0,1)$. We now state the following

Theorem 6. Denoting by $v(f)$ the number of changes of sign of $f(x)$ in $[0,1]$ we have the inequalities

$$
v\left(B_{n}\right) \leqq Z\left(B_{n}\right) \leqq v(f) .
$$

This result, an analogue of Theorem 1, can be derived as a special case from a general theorem of S. Karlin [3]. It admits, however, a very simple direct proof. Indeed, with $z=x /(1-x)$ for $0<x<1$, we have

$$
\frac{B_{n}(x)}{(1-x)^{n}}=\sum_{\nu=0}^{n} f\left(\begin{array}{l}
\nu \\
n
\end{array}\right)\left(\begin{array}{l}
n \\
\nu
\end{array}\right) z^{\prime}
$$

hence by Descartes' rule of signs

$$
Z\left(B_{n}\right)=Z\left(\frac{B_{n}(x)}{(1-x)^{n}}\right)=\underset{(0, \infty)}{Z}\left(\sum_{0}^{n} f\left(\begin{array}{c}
\nu \\
n
\end{array}\right)\left(\begin{array}{l}
n \\
\nu
\end{array}\right) z^{\nu}\right) \leqq v\left(f\left(\frac{\nu}{n}\right)\right) \leqq v(f) .
$$

8. The graphic behavior of the Bernstein polynomials. If we write $B_{n}(x)=B_{n}(x ; f)$ to indicate the dependence on $f(x)$, it is known that

$$
B_{n}(x ; A x+B)=A x+B .
$$

But then (7.1) implies that $B_{n}(x)-A x-B$ is the Bernstein polynomial of $f(x)-A x-B$. Now (7.2) implies the

Corollary 6. If $A x+B$ is an arbitrary linear function then

$$
Z\left(B_{n}(x)-A x-B\right) \leqq v(f(x)-A x-B) .
$$

Intersecting the graphs of $f(x)$ and $B_{n}(x)$ by appropriate straight lines $y=A x+B$, the inequality (8.2) furnishes a good deal of information concerning the shape of the graph of $B_{n}(x)$. Notice in particular the following 
Corollary 7. If $f(x)$ is convex in $[0,1]$, possibly discontinuous at the endpoints, but not linear in $[0,1]$, then

1. $B_{n}(x)$ is convex,

2. $B_{n}(x)>f(x)$ if $0<x<1$,

3. $B_{n}(0)=f(0), B_{n}(\mathcal{1})=f(1)$.

We may omit the simple proof based an Corollary 6 .

Observe that the relation $B_{n}^{\prime}(x ; f)=B_{n}\left(x ; f^{\prime}\right)$ is not valid. However a simple calculation shows that (7.1) implies

$$
\boldsymbol{B}_{n}^{\prime}(x)=n \sum_{0}^{n-1}\left(\begin{array}{c}
n-1 \\
\nu
\end{array}\right) \Delta f_{\nu} x^{\nu}(1-x)^{n-1-\nu}
$$

and

$$
B_{n}^{\prime \prime}(x)=n(n-1) \sum_{0}^{n-2}\left(\begin{array}{c}
n-2 \\
\nu
\end{array}\right) \Delta^{2} f_{\nu} x^{\nu}(1-x)^{n-2-\nu}
$$

where we have set

$$
\begin{gathered}
f_{\nu}=f\left(\frac{\nu}{n}\right), \\
\Delta f_{\nu}=f_{\nu+1}-f_{\nu}, \Delta^{2} f_{\nu}=f_{\nu+2}-2 f_{\nu+1}+f_{\nu} .
\end{gathered}
$$

(See Natanson [5], p. 179, fifth line from the bottom). The Theorems 3,4 and 5 have precise analogues as will now be shown with a minimum of details. The function classes $D_{1}$ and $D_{2}$ have analogues in the present situation and the numbers of sense-reversals and turn-reversals may again be defined by the relations

$$
\begin{array}{ll}
S(f)=\lim _{n \rightarrow \infty} v\left(\Delta f_{\nu}\right)=\sup _{n} v\left(\Delta f_{\nu}\right) & f \in D_{1}, \\
T(f)=\lim _{n \rightarrow \infty} v\left(\Delta^{2} f_{\nu}\right)=\sup _{n} v\left(\Delta^{2} f_{\nu}\right) & f \in D_{2},
\end{array}
$$

respectively.

As in the periodic case we obtain the following.

THEOREM 7. If $f(x) \in D_{1}$ then

$$
S\left(B_{n}\right)=v\left(B_{n}^{\prime}\right) \leqq S(f) .
$$

If $f(x) \in D_{2}$ then

$$
T\left(B_{n}\right)=v\left(B_{n}^{\prime \prime}\right) \leqq T(f)
$$

If $f(x)$ is odd about the point $x=\frac{1}{2}$, then $B_{n}(x)$ is found to share this property. As an analogue of Theorem 5 we have the following 
THEOREM 8. If $f(x)$ is odd about $x=\frac{1}{2}$, concave and non-negative in $\frac{1}{2} \leqq x \leqq 1$, positive in $\frac{1}{2}<x<1$, then also $B_{n}(x)$ is concave in $\left[\frac{1}{2}, 1\right]$ and

$$
0<B_{n}(x)<f(x) \text { if } \frac{1}{2}<x<1 .
$$

Indeed, let us first observe the following. Because of the invariance of linear functions expressed by (8.1), we may subtract from $f(x)$ the linear function whose graph is the chord joining the extreme points $(0, f(0))$ and $(1, f(1))$, without altering the assumptions on $f(x)$. Thus without loss of generality we may assume that $f(0)=f(1)=0$. From this point the proof is entirely similar to the proof of Theorem 5 in all details, including the use of the roof-functions. Finally notice that the equality is excluded in the second inequality (8.3). This is so because of the inequality (8.2) of Corollary 6 ; in the periodic case we only had the weaker analogue (5.2).

\section{APPENDIX II. A CONJECTURE ON POWER SERIES MAPPING A CIRCLE ONTO A CONVEX DOMAIN}

9. Sources and forms of the conjecture. As stated in the Introduction, a power series

$$
a_{1} z+a_{2} z^{2}+a_{3} z^{3}+\cdots+a_{n} z^{n}+\cdots=f(z)
$$

is said to belong to the class $K$, if it converges in the circle $|z|<1$ and maps this circle onto a convex domain. We say that the infinite sequence of complex numbers $\lambda_{1}, \lambda_{2}, \lambda_{3}, \cdots \lambda_{n}, \cdots$ is a convexity-preserving factor sequence if the series $\lambda_{1} a_{1} z+\lambda_{2} a_{2} z^{2}+\lambda_{3} a_{3} z^{3}+\cdots$ necessarily belongs to $K$ whenever (9.1) belongs to $K$. Let us apply such a factor sequence to the simplest power series belonging to $K$, to the geometric series

$$
z+z^{2}+z^{3}+\cdots=\frac{z}{1-z} .
$$

We obtain

$$
\lambda_{1} z+\lambda_{2} z^{2}+\lambda_{3} z^{3}+\cdots+\lambda_{n} z^{n}+\cdots ;
$$

if $\lambda_{1}, \lambda_{2}, \lambda_{3}, \cdots$ is a convexity-preserving factor sequence, the power series (9.3) must necessarily belong to $K$. We state the conjecture that this obvious necessary condition is also sufficient; that is, we formulate 
ConJeCtuRE I. If both power series

$$
\begin{gathered}
a_{1} z+a_{2} z^{2}+a_{3} z^{3}+\cdots \\
b_{1} z+b_{2} z^{2}+b_{3} z^{3}+\cdots
\end{gathered}
$$

belong to $K$, also

$$
a_{1} b_{1} z+a_{2} b_{2} z^{2}+a_{3} b_{3} z^{3}+\cdots
$$

belongs to $K .^{2}$

In view of Lemma 5 , the conjecture can be restated in other forms, equivalent to the first.

ConJECTURE II. If the power series

$$
a_{1} z+a_{2} z^{2}+a_{3} z^{3}+\cdots
$$

belongs to $K$ and

$$
b_{1} z+b_{2} z^{2}+b_{3} z^{3}+\cdots
$$

belongs to $\Sigma$, then

$$
a_{1} b_{1} z+a_{2} b_{2} z^{2}+a_{3} b_{3} z^{3}+\cdots
$$

belongs to $\Sigma$.

CONJECTURE III. If both power series

$$
\begin{aligned}
& a_{1} z+a_{2} z^{2}+a_{3} z^{3}+\cdots \\
& b_{1} z+b_{2} z^{2}+b_{3} z^{3}+\cdots
\end{aligned}
$$

belong to $\Sigma$, also

$$
\frac{a_{1} b_{1}}{1} z+\frac{a_{2} b_{2}}{2} z^{2}+\frac{a_{3} b_{3}}{3} z^{3}+\cdots
$$

belongs to $\Sigma$.

These three Conjectures I, II and III are completely equivalent, they stand and fall together. The third form brings out most clearly the relation to a conjecture that has been found, years ago and independently of each other, by two of our friends, Professor S. Mandelbrojt and Professor M. Schiffer, and which is published here with their permission :

2 One of the "intuitive sources" of the conjecture is the feeling that (9.2) plays a "leading role" in $K$, that it "sets the fashion." Which one of the two authors of this paper is the author of the conjecture will be disclosed if and when the conjecture is proved. 
Conjecture M. S. If both power series

$$
\begin{aligned}
& a_{1} z+a_{2} z^{2}+a_{3} z^{3}+\cdots \\
& b_{1} z+b_{2} z^{2}+b_{3} z^{3}+\cdots
\end{aligned}
$$

are "schlicht" in the unit circle, also

$$
\frac{a_{1} b_{1}}{1} z+\frac{a_{2} b_{2}}{2} z^{2}+\frac{a_{3} b_{3}}{3} z^{3}+\cdots
$$

is "schlicht" in the unit circle.

Whereas III is equivalent to I or II, it appears logically independent of MS. As far as obvious conclusions from the statements go, III could be true but MS false, or MS true yet III false, or both could be true or both false. Still, the conjectures are obviously related and their joint consideration may lead to various suggestions.

The Conjectures I, II and III are more "elementary" than MS and they are certainly more accessible; we succeeded in treating several of their particular cases and consequences.

10. Verification of the conjecture in some particular cases. We shall exhibit several particular series $\sum b_{n} z^{n}$ belonging to $K$ which, convoluted with an arbitrary series (9.1) belonging to $K$, generate a series $\sum a_{n} b_{n} z^{n}$ belonging to $K$.

(a) The polynomial (6.14) belongs to $K$. That its convolution with an arbitrary series belonging to $K$ necessarily belongs to $K$ is precisely what Theorem 2 asserts.

(b) If the series (9.1) belongs to $K$, it belongs, a fortiori, to $\Sigma$. Therefore, by Lemma 5, the series

$$
\frac{a_{1} z}{1}+\frac{a_{2} z^{2}}{2}+\frac{a_{3} z^{3}}{3}+\cdots
$$

belongs to $K$. This is another special case of Conjecture I; that the series

$$
\frac{z}{1}+\frac{z^{2}}{2}+\frac{z^{3}}{3}+\cdots=\log \frac{1}{1-z}
$$

maps the unit circle onto a convex domain follows from its relation to (9.2) and from Lemma 5 but this fact can also be established directly (see [9, vol. 1, p. 106, problem 114]).

(c) The result mentioned under (a) (Theorem 2) is due to the fact that the $V$-means are variation diminishing; cf. \$6. Any variation diminishing transformation on the circle leads to an analogous result, 
and so we obtain especially the following (cf. [4]). Let $g(z)$ be the product of $e^{-\gamma z^{2}}$, where $r \geqq 0$, with an entire function of genus 1 , all coefficients and all zeros of which are reat; then

$$
\sum_{1}^{\infty} \frac{z^{n}}{g(i n)}
$$

belongs to $K$, and, provided that (9.1) belongs to $K$, also

$$
\sum_{1}^{\infty} \frac{a_{n} z^{n}}{g(i n)}
$$

belongs to $K$. The term "entire function of genus 1 " is used here in the comprehensive sense, that is, it is supposed to include also entire functions of genus 0 and polynomials (but, obviously, not the identically vanishing polynomial); the case in which $g(z)$ reduces to $z$ was mentioned under (b).

(d) Let $p$ and $q$ denote two different given points on the unit circle $(|p|=|q|=1, p \neq q)$. Assume that (9.1) belongs to $K$ and let $z$ describe a circle concentric with, and interior to, the unit circle. Then $f(z)$ describes a convex curve of which $f(p z)-f(q z)$ represents a moving chord; as it is easy to see geometrically this chord turns all the time in the same sense. The argument of the complex number $f(p z)-f(q z)$ increases steadily. That is, the power series

$$
\frac{f(p z)-f(q z)}{p-q}=\sum_{1}^{\infty} a_{n} \frac{p^{n}-q^{n}}{p-q} z^{n}
$$

belongs to $\Sigma$ (maps the unit circle onto a star-shaped domain) and so, by Lemma 5 , the power series

$$
\sum_{n=1}^{\infty} \frac{a_{n}}{n} \frac{p^{n}-q^{n}}{p-q} z^{n}
$$

belongs to $K$ (cf. [6]). The series (10.1) is the convolution of (9.1) and of that particular case of (10.1) in which $a_{n}=1$; this particular series maps $|z|<1$ onto an infinite strip bounded by two parallels.

11. Verification of some consequences. In the foregoing, we have dealt mainly with form I of the conjecture, but now we shall consider its form III. We assume, therefore, that the function (9.1) belongs to the class $\Sigma$, that is, it maps the circle $|z|<1$ onto a star-shaped domain. We shall say that (9.1) is normalized if

$$
a_{1}=1 \text {. }
$$


(a) We are given an integer $n, n \geqq 2$. Let us consider the normalized functions of the class $\Sigma$ and let us seek one for which $\left|a_{n}\right|$ is a maximum. We leave aside the (easy) discussion of the existence and assume that (9.1) is such a function with maximum $\left|a_{n}\right|$. Now we apply Conjecture III with $b_{m}=a_{m}$ for $m=1,2,3, \cdots$; the resulting series is again normalized and so its $n$th coefficient cannot have an absolute value exceeding the maximum ; that is, $\frac{\left|a_{n}\right|^{2}}{n} \leqq\left|a_{n}\right|$, from which it follows that

$$
\left|a_{n}\right| \leqq n
$$

For series of the class $\Sigma$ this inequality is well known and easily established independently of the Conjecture III. And so our previous reasoning served only to enhance somewhat the plausibility of Conjecture III. Yet the same reasoning is also applicable to the Conjecture $M S$ and reveals one of the essential sources of this Conjecture.

(b) The function $f(z)$ belongs to the class $\Sigma$ if, and only if,

$$
\frac{z f^{\prime}(z)}{f(z)}=1+2 \alpha_{1} z+2 \alpha_{2} z^{2}+2 \alpha_{3} z^{3}+\cdots
$$

is regular in the circle $|z|<1$ and has there a positive real part. This will be the case if, and only if, the Hermitian form of the variables $z_{0}, z_{1}, \cdots, z_{n}$

$$
\sum_{k=0 l=0}^{n} \sum_{k-l}^{n} \alpha_{k} \bar{z}_{l}
$$

$\left(\alpha_{-\nu}=\bar{\alpha}_{\nu}\right.$, by definition) is positive (definite or semidefinite) for $n=1,2$, $3, \cdots$. This well known important necessary and sufficient condition is due to Carathéodory and Toeplitz. It can also be expressed in terms of the determinants

$$
\boldsymbol{A}_{n}=\left|\begin{array}{lllll}
1 & \alpha_{1} & \alpha_{2} & \cdots & \alpha_{n} \\
\alpha_{-1} & 1 & \alpha_{1} & \cdots & \alpha_{n-1} \\
\alpha_{-2} & \alpha_{-1} & 1 & \cdots & \alpha_{n-2} \\
\cdot & \cdot & \cdot & \cdot & \cdot \\
\alpha_{-n} & \alpha_{-n+1} & \alpha_{-n+2} & \cdots & 1
\end{array}\right| .
$$

Now (see (9.1)) the relation (11.2) can be written in the form

$$
a_{1} z+a_{2} z^{2}+a_{3} z^{3}+\cdots=a_{1} z \exp \left(\frac{2 \alpha_{1} z}{1}+\frac{2 \alpha_{2} z^{2}}{2}+\frac{2 \alpha_{3} z^{3}}{3}+\cdots\right)
$$

or in the form, 


$$
\frac{2 \alpha_{1} z}{1}+\frac{2 \alpha_{2} z^{2}}{2}+\frac{2 \alpha_{3} z^{3}}{3}+\cdots=\log \left(1+\frac{a_{2} z}{a_{1}}+\frac{a_{3} z^{2}}{a_{1}}+\cdots\right)
$$

and so we can express both $a_{n} / a_{1}$ as a polynomial in the $\alpha$ and $\alpha_{n}$ as a polynomial in the $a / a_{1}$ :

$$
\begin{aligned}
& \frac{a_{2}}{a_{1}}=2 \alpha_{1} \\
& \frac{a_{3}}{a_{1}}=\frac{\left(2 \alpha_{1}\right)^{2}+2 \alpha_{2}}{2 !} \\
& \frac{a_{4}}{a_{1}}=\frac{\left(2 \alpha_{1}\right)^{3}+2\left(2 \alpha_{3}\right)+3\left(2 \alpha_{1}\right)\left(2 \alpha_{2}\right)}{3 !} \\
& \ldots \\
& 2 \alpha_{1}=\frac{a_{2}}{a_{1}} \\
& 2 \alpha_{2}=-\frac{a_{2}^{2}-2 a_{1} a_{3}}{a_{1}^{2}} \\
& 2 \alpha_{3}=\frac{a_{2}^{3}-3 a_{1} a_{2} a_{3}+3 a_{1}^{2} a_{4}}{a_{1}^{3}}
\end{aligned}
$$

It would be easy to write down (11.7) or (11.8) for general $n$, but we shall not enter into details. Using (11.8) we could express the Hermitian form (11.3) and the determinant (11.4) in terms of the coefficients of the series (9.1) and doing so we wonld render more explicit the necessary and sufficient condition for the class $\Sigma$. Yet we postpone this consideration.

(c) Now consider, besides (9.1), two other power series with coefficients $b_{n}$ and $c_{n}$ respectively, and let $\beta_{n}$ and $\boldsymbol{B}_{n}$ be so linked to the $b$, and $\gamma_{n}$ and $\boldsymbol{C}_{n}$ so linked to the $c$, as $\alpha_{n}$ and $\boldsymbol{A}_{n}$ are to the $a$. Thus we have besides (11.5) (in all summations $n=1,2,3, \cdots$ )

$$
\sum b_{n} z^{n}=b_{1} z \exp \left(2 \sum \frac{\beta_{n} z^{n}}{n}\right), \quad \sum c_{n} z^{n}=c_{1} z \exp \left(2 \sum \frac{\gamma_{n} z^{n}}{n}\right)
$$

Set

$$
\frac{a_{n} b_{n}}{n}=c_{n}
$$

Now express $a_{n} / a_{1}$ in terms of the $\alpha$ from (11.7), and express analogously $b_{n} / b_{1}$ in terms of the $\beta$, then $c_{n} / c_{1}$ in terms of the $\alpha$ and $\beta$ from (11.10) 
and finally from relations analogous to (11.8), express $\gamma_{n}$ in terms of the $c / c_{1}$ and so in terms of the $\alpha$ and $\beta$. This leads to

$$
\begin{aligned}
\gamma_{1}= & \alpha_{1} \beta_{1} \\
3 \gamma_{2}= & \alpha_{2} \beta_{2}+2 \alpha_{2} \beta_{1}^{2} \\
& +2 \alpha_{1}^{2} \beta_{2}-2 \alpha_{1}^{2} \beta_{1}^{2} \\
6 \gamma_{3}= & \alpha_{3} \beta_{3}+3 \alpha_{3} \beta_{1} \beta_{2}+2 \alpha_{3} \beta_{1}^{3} \\
& +3 \alpha_{1} \alpha_{2} \beta_{3}+3 \alpha_{1} \alpha_{2} \beta_{1} \beta_{2}-6 \alpha_{1} \alpha_{2} \beta_{1}^{3} \\
& +2 \alpha_{1}^{3} \beta_{3}-6 \alpha_{1}^{3} \beta_{1} \beta_{2}+4 \alpha_{1}^{3} \beta_{1}^{3}
\end{aligned}
$$

Not all details of the general formula for $\gamma_{n}$ are obvious; a few features will be discussed under (e). The determinant $\boldsymbol{C}_{n}$ (expressed in terms of the $\gamma$ as $\boldsymbol{A}_{n}$ is in terms of the $\alpha$, cf. (11.4)) becomes by virtue of (11.11) a polynomial in the $\alpha, \bar{\alpha}, \beta$ and $\bar{\beta}$. By the theory of Caratheodory and Toeplitz, Conjecture III is equivalent to the following.

CONJECTURE IV. The $2 n$ inequalities

$$
\begin{gathered}
\boldsymbol{A}_{1}>0, \boldsymbol{A}_{2}>0, \cdots, \boldsymbol{A}_{n}>0, \\
\boldsymbol{B}_{1}>0, \boldsymbol{B}_{2}>0, \cdots, \boldsymbol{B}_{n}>0,
\end{gathered}
$$

imply the $n$ inequalities

$$
\boldsymbol{C}_{1}>0, \boldsymbol{C}_{2}>0, \cdots, \boldsymbol{C}_{n}>0
$$

and this holds for $n=1,2,3, \ldots$.

This formulation excludes the case of equality in all the $3 n$ inequalities considered. This is due to the fact that, without loss of generality, we may suppose $\Sigma a_{n} z^{n}$ and $\Sigma b_{n} z^{n}$ regular in $|z| \leqq 1$.

(d) The case $n=1$ of Conjecture IV is trivial. In fact, if we assume that the series are normalized, see (11.1), and introduce the coefficients of the mapping functions, see (11.8), the statement that we have to prove reduces to this:

$$
\begin{array}{lc}
\text { The inequalities } & \left|a_{2}\right|<2,\left|b_{2}\right|<2 \\
\text { imply } & \frac{\left|a_{2} b_{2}\right|}{2}<2
\end{array}
$$

which is obvious.

(e) The case $n=2$ of Conjecture IV was first established by Dr. G. A. Hummel and can be proved as follows.

We take the series as normalized, see (11.1), and set

$$
a_{2}=a, a_{3}=A, b_{2}=b, b_{3}=B ;
$$


we suppose, without loss of generality, that $a \geqq 0, b \geqq 0$. We have to show :

The two inequalities

$$
\left|A-\frac{3 a^{2}}{4}\right|<1-\frac{a^{2}}{4},\left|B-\frac{3 b^{2}}{4}\right|<1-\frac{b^{2}}{4}
$$

imply

$$
\left|\frac{A B}{3}-\frac{3 a^{2} b^{2}}{16}\right|<1-\frac{a^{2} b^{2}}{16}
$$

(The first inequality (11.12) results from the condition $\boldsymbol{A}_{2}>0$, see (11.4), by virtue of (11.8); it implies $a<2$, and so the condition $\boldsymbol{A}_{1}>0$.)

Let

$$
A=\frac{3 a^{2}}{4}+u, \quad B=\frac{3 b^{2}}{4}+v .
$$

By the hypothesis (11.12) of the theorem that we are about to prove

$$
|u|<1-\frac{a^{2}}{4}, \quad|v|<1-\frac{b^{2}}{4} .
$$

We derive from (11.14) and (11.15)

$$
\begin{aligned}
& A B=\frac{9 a^{2} b^{2}}{16}+\frac{3 a^{2}}{4} v+\frac{3 b^{2}}{4} u+u v \\
& \left|A B-\frac{9 a^{2} b^{2}}{16}\right|<\frac{3 a^{2}}{4}\left(1-\frac{b^{2}}{4}\right)+\frac{3 b^{2}}{4}\left(1-\frac{a^{2}}{4}\right)+\left(1-\frac{a^{2}}{4}\right)\left(1-\frac{b^{2}}{4}\right) .
\end{aligned}
$$

We assert that

$$
1+\frac{a^{2}}{2}+\frac{b^{2}}{2}-\frac{5 a^{2} b^{2}}{16}<3-\frac{3 a^{2} b^{2}}{16}
$$

in fact, this follows from $a<2, b<2$, since it is equivalent to

$$
\left(1-\frac{a^{2}}{4}\right)\left(1-\frac{b^{2}}{4}\right)>0 \text {. }
$$

The right hand side of (11.16) is equal to the left hand side of (11.17), and so the combination of these two inequalities immediately yields the desired conclusion (11.13).

(e) We consider now the expression of $\gamma_{n}$ in terms of the $\alpha$ and 
$\beta$ for general $n$; for the cases $n=1,2,3$, see (11.11). The procedure that led us to (11.11) shows that $\gamma_{n}$ is a polynomial in $\alpha_{1}, \alpha_{2}, \cdots, \alpha_{n}$, $\beta_{1}, \beta_{2}, \cdots, \beta_{n}$ with rational coefficients. Obviously, by virtue of (11.10), $\gamma_{n}$ is symmetric in the $\alpha$ and $\beta$. If we substitute $\rho z$ for $z$ in (11.5) or, which is the same, we change $\alpha_{n}$ into $\rho^{n} \alpha_{n}$ and $a_{n}$ into $\rho^{n} a_{n}$, there results a change, see again (11.10), of $c_{n}$ into $\rho^{n} c_{n}$ and of $\gamma_{n}$ into $\rho^{n} \gamma_{n}$; therefore, $\gamma_{n}$ must be an isobaric polynomial in the $\alpha$ of weight $n$. Finally, $r_{n}$ must be of the form

$$
\gamma_{n}=\sum_{k=1 l=1}^{p} \sum_{k l}^{p} j_{k l}^{(n)} A_{k} B_{l}
$$

where

$p=p(n)$ is the number of partitions of the integer $n$,

$A_{1}, A_{2}, \cdots, A_{p}$ are the products of powers of weight $n$ of $\alpha_{1}, \alpha_{2}, \cdots$, $\alpha_{n}$, ordered lexicographically so that

$$
A_{1}=\alpha_{n}, A_{2}=\alpha_{n-1} \alpha_{1}, \cdots A_{p}=\alpha_{1}^{n} .
$$

Generally $A_{k}$ is of the form

$$
A_{k}=\alpha_{1}^{k_{1}} \alpha_{2^{2}}^{k_{2}} \cdots \alpha_{n^{k}}^{k_{n}}
$$

its weight $1 k_{1}+2 k_{2}+3 k_{3}+\cdots+n k_{n}=n$.

$B_{1}, B_{2}, \cdots, B_{p}$ are analogously expressed in terms of $\beta_{1}, \beta_{2}, \cdots, \beta_{n}$, and $j_{i k}^{(n)}$ are rational numbers, $j_{i k}^{(n)}=j_{k i}^{(n)}$.

For example $p(4)=5$ and, for $n=4$

$$
A_{1}=\alpha_{4}, A_{2}=\alpha_{3} \alpha_{1}, A_{3}=\alpha_{2}^{2}, A_{1}=\alpha_{2} \alpha_{1}^{2}, A_{i}=\alpha_{1}^{4},
$$

the $B$ are analogously defined and the matrix of the $j_{i k}^{(4)}$ results from

$\begin{array}{rrrrr}9 & 24 & 9 & 36 & 12 \\ 24 & 24 & 24 & -24 & -48 \\ 9 & 24 & -1 & -4 & -28 \\ 36 & -24 & -4 & -136 & 128 \\ 12 & -48 & -28 & 128 & -64\end{array}$

if each of the 25 numbers displayed is divided by 90 .

We cannot exhibit the law of the dependence of $j_{i k}^{(n)}$ on $n$ in some obviously useful manner, but we note here one property. If $\beta_{n}=1$ it is easily seen from (11.9) that $b_{n} / b_{1}=n$ and, therefore, by (11.10) $c_{n} / c_{1}=$ $a_{n} / a_{1}$ and so finally

$$
\gamma_{n}=\alpha_{n}
$$

for any choice of the $\alpha_{n}$; this must be compatible with (11.18) and so, since $B_{1}=B_{2}=\cdots=B_{p}=1$, by our choice of the $\beta$, 


$$
\sum_{l=1}^{p} j_{k l}^{(n)}=\left\{\begin{array}{l}
1 \text { for } k=1 \\
0 \text { for } k=2,3, \cdots, p
\end{array}\right.
$$

(f) The system of $n$ complex numbers $\left(\alpha_{1}, \alpha_{2}, \cdots, \alpha_{n}\right)$, for which we shall also use the more concise notation $(\alpha)$, determines a point in $2 n$-dimensional Euclidean space. A point $(\alpha)$ belongs to the coefficientdomain if, and only if, it corresponds by virtue of (11.5) to the initial terms of a power series of the class $\Sigma$. The most remarkable boundary point of the coefficient domain is the "Koebe-point" which corresponds to the function

$$
z+2 z^{2}+3 z^{3}+\cdots=z(1-z)^{-2} .
$$

Our aim is to show that, for any given $n$, Conjecture IV is true for two interior points of the coefficient domain which are sufficiently close to the Koebe-point.

Let us choose two arbitrary points $(u)$ and $(v)$ in the interior or the coefficient domain. That is, (cf. under $(b)$ ) both Hermitian forms

$$
\sum \sum u_{k-l} z_{k} \bar{z}_{l}, \quad \sum \sum v_{k-\imath} z_{k} \bar{z}_{l}
$$

are positive definite. Let $\alpha, \beta$ and $\varepsilon$ denote positive numbers ; $\alpha$ and $\beta$ are arbitrary and $\varepsilon$ so small that $\alpha \varepsilon<1, \beta \varepsilon<1$. The coefficient domain is convex. Therefore, if we set

$$
\alpha_{\nu}=(1-\varepsilon \alpha)+\varepsilon \alpha u_{\nu}, \quad \beta_{\nu}=(1-\varepsilon \beta)+\varepsilon \beta v_{\nu}
$$

for $\nu=0, \pm 1, \pm 2, \cdots, \pm n$, the points $(\alpha)$ and $(\beta)$ are in the interior of the coefficient domain. If $A_{k}$ is given by (11.20)

$$
A_{k}=1+\varepsilon \alpha \tilde{u}_{k}+O\left(\varepsilon^{2}\right)
$$

where

$$
\tilde{u}_{k}=k_{1}\left(u_{1}-1\right)+k_{2}\left(u_{2}-1\right)+\cdots+k_{n}\left(u_{n}-1\right)
$$

and $O\left(\varepsilon^{2}\right)$ denotes a quantity of order not exceeding $\varepsilon^{2}$ when $\varepsilon$ tends to 0 . There is a similar expression for $B_{l}$ and finally, by (11.21),

$$
\begin{aligned}
\gamma_{n} & =\sum_{k=1 l=1}^{p} \sum_{k l}^{p} j_{k l}^{(n)} A_{k} B_{l} \\
& =\sum_{k=1 l=1}^{p} \sum_{k l}^{p} j_{k l}^{(n)}\left[1+\varepsilon \alpha \tilde{u}_{k}+\varepsilon \beta \tilde{v}_{l}\right]+O\left(\varepsilon^{2}\right) \\
& =1+\varepsilon \alpha \tilde{u}_{1}+\varepsilon \beta \tilde{v}_{1}+O\left(\varepsilon^{2}\right) \\
& =1+\varepsilon \alpha\left(u_{n}-1\right)+\varepsilon \beta\left(v_{n}-1\right)+O\left(\varepsilon^{2}\right) .
\end{aligned}
$$

By virtue of (11.24) 


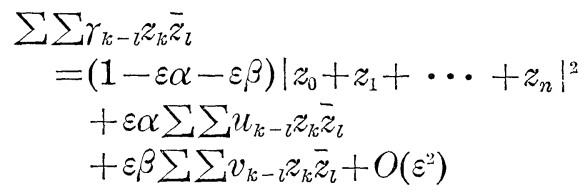

and this Hermitian form is definite positive for sufficiently small $\varepsilon$, since the forms (11.22) are definite positive. With this, we have proved another infinitesimal part of Conjecture IV.

\section{REFERENCES}

1. L. Fejér, Gestaltliches ueber die Partialsummen und ihre Mittelwerte bəi der Fourizrreine und der Potenzreihe, Z. f. angew. Math. und Mech., 13 (1933), 80-88.

2. A. Hurwitz, Mathematische Werke, Vol. I.

3. S. Karlin, Pólya-type distributions II. Ann. Math. Stat., 28 (1957), 281-308.

4. J. C. Mairhuber, I. J. Schoenberg and R. Williamson, Variation diminishing transformations on the circle, to be published.

5. I. P. Natanson, Konstruktive Funktionentheorie (German translation from Russian), Berlin, 1955.

6. Z. Nehari, Conformal Mapping, New York, 1952.

7. G. Pólya, Ueber einige Verallgemeinerungen der Descarteschen Zeichenregel, Archiv Math. Phys. III. Reihe, 23 (1914), 22-32.

8. G. Pólya and N. Wiener, On the oscillation of the derivatives of a periodic functicn, Trans. Amer. Math. Soc., 52 (1942), 249-256.

9. G. Pólya and G. Szegö, Aufgaben und Lehrsaetze aus der Analysis, Berlin, 1925.

10. I. J. Schoenberg, Smoothing operations and thoir geizerating functions, Bull. Amer. Math. Soc., 59 (1953), 199-230.

11. E. Study, Vorlesungen ueber ausgewaehlte Gegenstaende der Geometrie, Second Part, Leipzig and Berlin, 1913.

12. J. J. Sylvester, Collected mathematical papers, Vol. II, Cambridge, 1908.

13. G. Szegö, Zur Theorie der schlichten Abbildungen, Math. Ann. 100 (1929), 88-211. 14. Ch. J. de la Vallée Poussin, Sur l'approximation des fonctions d'une variable réelle et de leurs dérivées par des polynomes et des suites limitées de Fourier, Bull. Acad. de Belgique (classe des sciences), 3 (1908), 193-254.

STANFORD UNIVERSITY

The University of Pennsylvania 


\section{PACIFIC JOURNAL OF MATHEMATICS}

\section{EDITORS}

\section{H. L. Royden}

Stanford University

Stanford, California

\section{R. A. Beaumont}

University of Washington

Seattle 5 , Washington

\author{
A. L. Whiteman
}

University of Southern California

Los Angeles 7, California

E. G. Straus

University of California

Los Angeles 24, California

\section{ASSOCIATE EDITORS}
E. F. BECKENBACH
A. HORN
L. NACHBIN
G. SZEKERES
C. E. BURGESS
V. GANAPATHY IYER
I. NIVEN
F. WOLF
M. HALL
R. D. JAMES
T. G. OSTROM
E. HEWITT
M. S. KNEBELMAN
M. M. SCHIFFER
K. YOSIDA

\section{SUPPORTING INSTITUTIONS}

\author{
UNIVERSITY OF BRITISH COLUMBIA \\ CALIFORNIA INSTITUTE OF TECHNOLOGY \\ UNIVERSITY OF CALIFORNIA \\ MONTANA STATE UNIVERSITY \\ UNIVERSITY OF NEVADA \\ OREGON STATE COLLEGE \\ UNIVERSITY OF OREGON \\ UNIVERSITY OF SOUTHERN CALIFORNIA
}

\author{
STANFORD UNIVERSITY \\ UNIVERSITY OF UTAH \\ WASHINGTON STATE COLLEGE \\ UNIVERSITY OF WASHINGTON \\ * * * * \\ AMERICAN MATHEMATICAL SOCIETY \\ CALIFORNIA RESEARCH CORPORATION \\ HUGHES AIRCRAFT COMPANY \\ THE RAMO-WOOLDRIDGE CORPORATION
}

Mathematical papers intended for publication in the Pacific Journal of Mathematics should be typewritten (double spaced), and the author should keep a complete copy. Manuscripts may be sent to any of the editors. All other communications to the editors should be addressed to the managing editor, E. G. Straus at the University of California, Los Angeles 24, California.

50 reprints per author of each article are furnished free of charge; additional copies may be obtained at cost in multiples of 50 .

The Pacific Journal of Mathematics is published quarterly, in March, June, September, and December. The price per volume (4 numbers) is $\$ 12.00$; single issues, $\$ 3.50$. Back numbers are available. Special price to individual faculty members of supporting institutions and to individual members of the American Mathematical Society: $\$ 4.00$ per volume; single issues, $\$ 1.25$.

Subscriptions, orders for back numbers, and changes of address should be sent to Pacific Journal of Mathematics, 2120 Oxford Street, Berkeley 4, California.

Printed at Kokusai Bunken Insatsusha (International Academic Printing Co., I.td.), No. 10, 1-chome, Fujimi-cho, Chiyoda-ku, Tokyo, Japan.

PUBLISHED BY PACIFIC JOURNAL OF MATHEMATICS, A NON-PROFIT CORPORATION

The Supporting Institutions listed above contribute to the cost of publication of this Journal, but they are not owners or publishers and have no responsibility for its content or policies. 


\section{Pacific Journal of Mathematics}

\section{Vol. 8, No. 2 \\ April, 1958}

John Herbert Barrett, Second order complex differential equations with a real independent variable ............................ 187

Avner Friedman, Remarks on the maximum principle for parabolic equations and its applications ......................... 201

Richard Robinson Goldberg, An inversion of the Stieltjes transform ....... 213

Olavi Hellman, On the periodicity of the solution of a certain nonlinear integral equation .................................. 219

Gilbert Helmberg, A theorem on equidistribution on compact groups...... 227

Lloyd Kenneth Jackson, Subfunctions and the Dirichlet problem ......... 243

Naoki Kimura, The structure of idempotent semigroups. I ............ 257

Stephen Kulik, A method of approximating the complex roots of equations........................................ 277

Ancel Clyde Mewborn, A note on a paper of L. Guttman.............. 283

Zeev Nehari, On the principal frequency of a membrane ............ 285

G. Pólya and I. J. Schoenberg, Remarks on de la Vallée Poussin means and convex conformal maps of the circle ...................... 295

B. M. Stewart, Asymmetry of a plane convex set with respect to its centroid .......................................... 335

Hans F. Weinberger, Lower bounds for higher eigenvalues by finite difference methods

Edwin Weiss and Neal Zierler, Locally compact division rings ......... 369

Bertram Yood, Homomorphisms on normed algebras ................. 373 